\title{
Acceptance Probabilities for a Sampling Procedure Based on the Mean and an Order Statistic
}

\author{
Mary C. Croarkin* and Grace L. Yang * * \\ Nationol Bureau of Standards, Washington, DC 20234 \\ October 13, 1982
}

\begin{abstract}
A dual acceptance criterion based on the sample mean and an extreme order is used in many inspection procedures. Computation of the acceptance probability for such a dual criterion is investigated. An approximation and a lower bound to the acceptance probability are derived and are applicable to any continuous distribution. In addition. the connection between this dual criterion and hypothesis testing of scale and location parameters is studied. In the case of the exponential distribution the exact evaluation of the acceptance probability yields the power of the test.
\end{abstract}

Key words: acceptance probability; compliance sampling: dual acceptance criteria; mixed sampling plan; order statistics; statistical methods.

\section{TABLE OF CONTENTS}

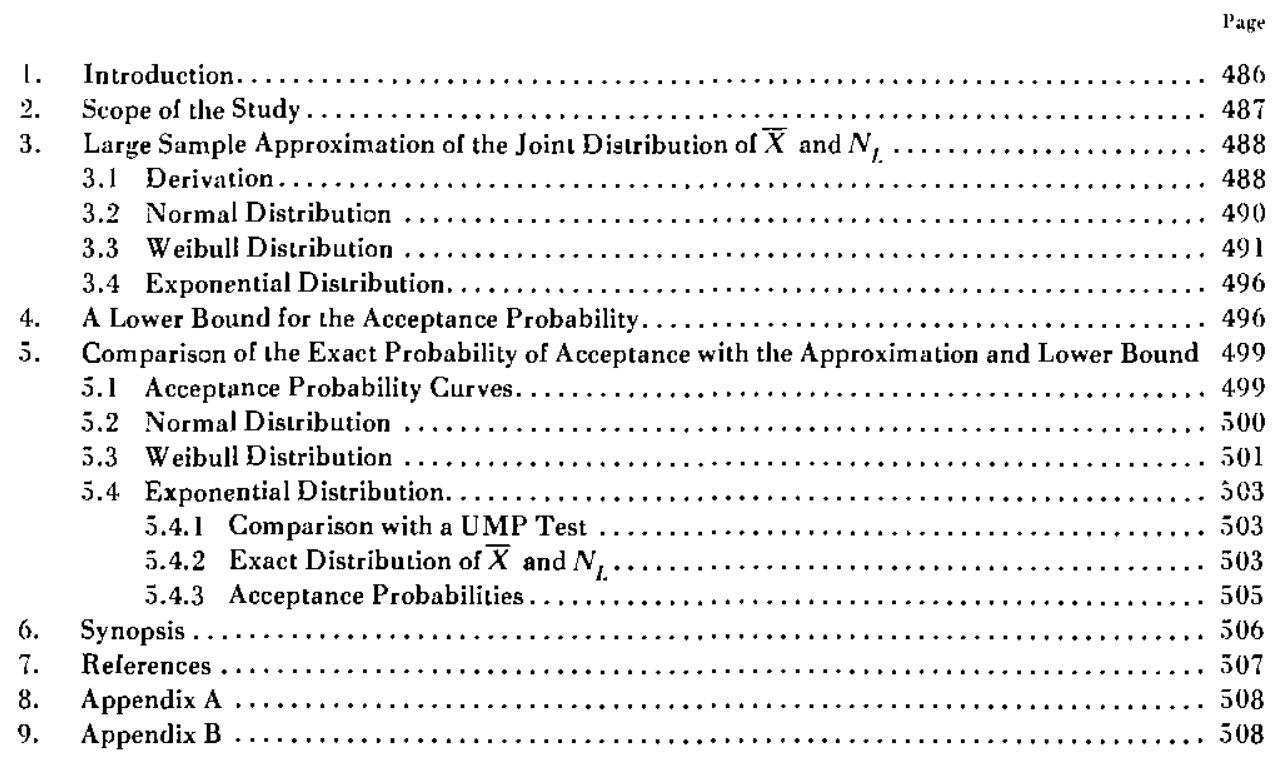

*Center for Applied Mathematics, National Engineering Laboratory.

**Center for Applied Mathematics, National Engineering Laboratory and the University of Maryland, College Park. MD. 


\section{Introduction}

Suppose that a random sample of size $n$ from a lot is measured with respect to a particular variable and that the acceptance or rejection of the lot depends upon whether or not the measurements satisfy certain criteria. "Lot" can refer to a group of individual items or to a specified amount of material which can be sampled randomly.

There is widespread interest in sampling procedures that specify acceptance criteria involving the sample mean and a proportion of defectives in the sample [1], [4], [5]. [9], [11] and [14]. ${ }^{1}$ Such a sampling procedure might specify that the lot is to be accepted only if the sample mean is greater than a value $\mu_{0}$, say, and if no more than a specified percentage of the sample is less than a lower limit $L$. The purpose of a dual acceptance criterion is to ensure, for example, that the lot is at least a stated amount. $\mu_{0}$, of the specified variable on the average and that the number of so called "defectives" or items that violate the lower limit is controlled. Obviously, depending on the application, the acceptance criteria can be specified in the opposite direction; i.e., the lot is to be accepted only if the sample mean is less than $\mu_{0}$ and at least a certain percentage of the sample is greater than an upper limit $U$.

Specifically, let $X_{1}, \cdots, X_{n}$ be a random sample of $n$ measurements, and let $X_{[j !} \leqslant \cdots \leqslant X_{[n]}$ be the corresponding order statistics. It is assumed that the random variables $X_{1}, \cdots, X_{n}$ are independent and identically distributed (i.i.d.) with a probability density function $f(x)$, and that the $X_{j}$ have finite mean $\mu$ and variance $\sigma^{2}$. Let $X$ be the sample mean and $N_{L}$ be the number of defectives or measurements having values smaller than the specified (lower) limit $L$.

The sampling procedure to be considered is such that the lot is accepted whenever

$$
\left[\bar{X} \geqslant \mu_{0} \text { and } N_{\mathrm{L}} \leqslant k\right]
$$

where $\mu_{0}$ and $k$ are specified in the sampling plan.

In terms of the order statistics, $\{1.1)$ is equivalent to the criterion

$$
\left[\bar{X} \geqslant \mu_{0} \text { and } X_{(k+1)}>L\right]
$$

and the probability of accepting the lot is defined to be

$$
P_{n}=P\left[\bar{X} \geqslant \mu_{0}, N_{L} \leqslant k\right]
$$

The sampling procedure discussed above is a mixed variables-attributes acceptance criterion based on one sample. There are various ways of designing a mixed sampling plan. The type studied by Schilling and Dodge [19] is a double sampling procedure involving variables inspection in the first sample. If the variables inspection does not lead to acceptance, a second sample is taken and an attribute inspection is conducted on the combined samples. In their work. Schilling and Dodge assume a normal distribution with unknown mean and known variance.

We concentrate on a single sample plan where both the variables inspection as specified by the sample mean and attributes inspection as specified by $k$, the number of allowable defectives, are conducted on the same sample. This causes difficulties in the computation of the acceptance probabilities because of the lack of independence of the sample mean and the order statistics.

Investigations, of which we are aware, into the statistical properties of sampling procedures of this type assume a normal distribution with unknown mean and known variance. For instance in a compliance sampling application, Weed [21] simulates a two-stage procedure used in specifications for the thickness of paving material in which both stages involve a variable and an attribute inspection. Elder and Muse [8] develop a large sample approximation for the acceptance probability used in U.S. Department of Agriculture inspection procedures (1.3) and compare the approximation to an exact numerical procedure.

\footnotetext{
'Figures in brackets indicate literature references at the end of this paper.
} 
It is noted that the dual sampling criterion leads to an acceptance region for testing hypotheses concerning the mean $\mu$ and the probability of item defectiveness simultaneously. The probability of a defective is defined to be $p=P[X \leqslant L]$. The acceptance region in (1.1) or (1.2) may be used for testing the null hypothesis

$$
H_{0}: \mu=\mu^{*} \text { and } p=p^{*}
$$

versus the alternatives

$$
H_{1}: \mu<\mu^{*} \text { or } p>p^{*}
$$

Through reparametrization, these hypotheses may be formulated in terms of the location and scale parameters. Evidently, this depends on the properties of the distribution under consideration.

In the case of the normal distribution $N\left(\mu, \sigma^{2}\right)$, the probability of a defective is

$$
p=\Phi\left(\frac{L-\mu}{\sigma}\right)
$$

where

$$
\Phi(z)=\frac{1}{\sqrt{2 \pi}} \int_{-\infty}^{z} \exp \left\{-u^{2} / 2\right\} d u .
$$

Thus,

$$
\sigma=(L-\mu) / \Phi^{-1}(p) .
$$

Consequently, $\mu=\mu^{*}$ and $p=p^{*}$ if and only if

$$
\mu=\mu^{*} \text { and } \sigma=\sigma^{*}=\left(L-\mu^{*}\right) / \Phi^{-1}\left(p^{*}\right) .
$$

Accordingly, the hypothesis testing problem in (1.4) becomes that of testing

$$
H_{0}: \mu=\mu^{*} \text { and } \sigma=\sigma^{*}
$$

versus

$$
H_{1}: \mu<\mu^{*} \text { or } \sigma<\frac{L-\mu}{\Phi^{-1}\left(p^{*}\right)}
$$

Perusal of the literature turned up very few papers that are directly related to a joint test of the location and scale parameters. Eisenberger [7] develops an asymptotic joint test for the mean and variance of a normal distribution based on a quantile. Perng [18] develops a joint test for the location and scale parameters of an exponential distribution based on Fisher's method of combining two test statistics. Anderson [2] discusses the likelihood ratio test for simultaneously testing the mean and variance in multivariate normal distributions; both one-sample and $k$-sample problems are considered. In a recent paper, Perlman [17] shows that the likelihood ratio test is unbiased. None of these papers discusses the computation of acceptance probabilities under alternative hypotheses. Also, unlike (1.7), the alternatives in the quoted papers are rectangular regions.

\section{Scope of the Study}

It is our intention to investigate the acceptance probability of a dual sampling procedure from several aspects. The investigations are carried out for the normal distribution because of its im- 
portance in acceptance sampling and for the exponential and Weibull distributions because of their application in modeling the life span distribution.

First, in section 3, we derive a large sample approximation $P_{a}$ for the acceptance probability $P_{n}$. This is achieved by deriving the asymptotic joint distribution of $\sqrt{n}(\bar{X}-\mu) / \sigma$ and $\left(N_{L}-n p\right) /(n p$ $(1-p)^{3 / 2}$ as the sample size approaches infinity. This approximation method applies to any distribution. We illustrate its use in the normal, Weibull. and exponential distributions. The results as given in sections $3.1,3.2$, and 3.3 are compared with a simulation study.

In section 4 a lower bound $\underline{P}$ is established for $P_{n}$ that amounts to assuming the independence of the sample mean and the $k^{\text {th }}$ order statistic. This lower bound for finite samples provides some information ou the accuracy of the approximation. We attempt to determine under what conditions the approximation $P_{a}$ is a significant improvement over the lower bound. In this connection one notes that a large sample approximation $P_{a}$ is derived by normalizing the sample mean as $\sqrt{n}(\bar{X}-\mu) / o$ and the number of defectives in the sample as $\left(N_{\mathrm{L}}-n p\right) /(n p(1-p))^{1 / 2}$. If, instead, we convert $N_{L}$ to an order statistic $X_{(k)}$ and consider $X_{(k)}$ (or $X_{(n-k)}$ as an extreme statistic, the normalized sample mean $\sqrt{n}(\bar{X}-\mu)$ and $X_{(k)}$ (or equivalently $X_{(n-k)}$ ) are asymptotically independent (The proof is given in appendix B|. This suggests that $\underline{P}$ serves as a possible approximation to $P_{n}$ when $n$ is large and $k$ is small.

In other words, when comparing $P_{s}$ and $\underline{P}$, one should keep in mind the relationship between $k$ and $n$; namely, the ratio $k / n$. In the case of $P_{a}$ we have $N_{L} / n \rightarrow p$ and in the case of an extreme statistic we have $k / n \rightarrow 0$ as $n \rightarrow \infty$. Clearly, one would expect that the lower bound $\underline{P}$ may be a reasonable approximation when $k$ is relatively small compared with $n$. This is indeed confirmed in our numerical study in section 4. The numerical studies show that $P_{a}$ is comparable to $\underline{P}$ for small $k / n$ and superior to $\underline{P}$ for larger values of $k / n$.

Finally, in section 5 the acceptance probabilities are approximated for the normal and Weibull distributions using a procedure proposed by Pearson and Hartley [16]. The exact acceptance probabilities curves are computed for the exponential distribution.

\section{Large Sample Approximation of the Joint Distribution of $\bar{X}$ and $N_{i}$.}

\subsection{Derivation}

Let $X_{1}, \cdots, X_{n}$ be a random sample from the lot with pdf $f(x)$. Assume that $X_{j}$ has a finite mean $\mu$ and variance $\sigma^{2}$.

Introducing indicator random variables $I_{j}$,

where

$$
I_{j}=\left\{\begin{array}{l}
1 \text { if } X_{j} \leqslant L \\
0 \text { if } X_{j}>L
\end{array}\right.
$$

and letting the probability that an item violates the lower specification limit $L$ be

$$
p=P\left[X_{j} \leqslant L\right]
$$

we can write the number of lunitl lower limit violations $N_{L}$ in the sample as

$$
N_{L}=\sum_{j=1}^{n} I_{j} .
$$

Note that $N_{L}$ has a binominal distribution $B(n, p)$, and the event $\left[N_{L} \leqslant k\right]$ is equivalent to the event $\left[X_{(k+1)}>L\right]$. In order to develop an approximation formula for the acceptance probability

$$
P_{n}=P\left[\bar{X} \geqslant \mu_{0}, N_{L} \leqslant k\right],
$$


we consider random variables $W_{n}$ and $Y_{n}$ defined as

$$
\text { and } \begin{aligned}
W_{n} & =n^{1 / 2}(\bar{X}-\mu) / \sigma \\
Y_{n} & =\left(N_{L}-n p\right) /(n p(1-p))^{1 / 2} .
\end{aligned}
$$

Let $\left(W_{n}, Y_{n}\right)$ be a row vector. We prove the following result.

THEOREM 3.1. As $\mathrm{n}->\infty$, the random vector $\left(\mathrm{W}_{n}, \mathrm{Y}_{\mathrm{n}}\right)^{\prime}$ converges in distribution to a bivariate normal distribution with mean $(0,0)^{\prime}$ and covariance matrix

$$
\Sigma=\left(\begin{array}{ll}
1 & e \\
e & 1
\end{array}\right)
$$

where

$$
\varrho=E\left(X_{j}-\mu\right) I_{j} / o(p(1-p))^{1 / 2} .
$$

Proof: Let $t_{1}$ and $t_{2}$ be arbitrarily chosen but fixed real numbers. Form the linear combination of $W_{n}$ and $Y_{n}, t_{1} W_{n}+t_{2} Y_{n}$.

Direct computation and application of the central limit theorem give

$$
t_{1} W_{n}+t_{2} Y_{n} \quad \stackrel{D}{\rightarrow} N\left(0, t_{1}^{2}+t_{2}^{2}+t_{1} t_{2} \varrho\right) \text { as } n \rightarrow \infty
$$

It then follows from application of the Cramer-Wold device that

$$
\left(\begin{array}{c}
W_{n} \\
Y_{n}
\end{array}\right) \stackrel{D}{\rightarrow} \quad N\left(\left(\begin{array}{l}
0 \\
0
\end{array}\right), \quad \Sigma\right) \text { as } n \rightarrow \infty
$$

where $\Sigma$ is given in 13.1 .5 ).

Making use of the asymptotic distribution in Theorem 3.1, we note from (3.1.4) that

$$
\begin{array}{cc}
\bar{X}= & n^{-1 / 2} \sigma W_{n}+\mu \\
& \text { and } \\
N_{L}= & (n p(1-p))^{1 / 2} Y_{n}+n p .
\end{array}
$$

Thus the random vector $\left(\bar{X}, N_{L}\right)^{\prime}$ has asymptotically a bivariate normal distribution with mean and covariance matrix $\Gamma$ given by

$$
\left(\begin{array}{l}
\mu \\
n p
\end{array}\right) \text { and } \Gamma=\left(\begin{array}{ll}
\frac{\sigma^{2}}{n} & E\left(X_{j}-\mu\right) I_{j} \\
E\left(X_{j}-\mu\right) I_{\mathrm{j}} & n p(1-p)
\end{array}\right)
$$

respectively.

For convenience in computation, write the acceptance probability $P_{n}$ as

$$
\begin{gathered}
P_{n}=P\left[\bar{X} \geqslant \mu_{0}\right]-P\left[\bar{X} \geqslant \mu_{0}, N_{L}>k\right] \\
=P\left[\bar{X} \geqslant \mu_{0}\right]-P\left[W_{n} \geqslant \sqrt{n}\left(\mu_{0}-\mu\right) / o, Y_{n}>\left(\left.n p(1-p)\right|^{-1 / 2}(k-n p)\right] .\right.
\end{gathered}
$$


Making use of (3.1.7) and the continuity correction factor 0.5 for the random variable $N_{L}$, we see that for sufficiently large $n, P_{n}$ may be approximated by

$$
P_{u}=\frac{I}{\sqrt{2 \pi}} \int_{a}^{\infty} \exp \left(-z^{2} / 2\right) d z-\int_{a}^{\infty} \int_{b}^{\infty} g(x, y, \varrho) d x d y
$$

where

$$
\begin{gathered}
\left.a=\sqrt{n} \mid \mu_{0}-\mu\right) / \sigma, \\
b=(n p(1-p))^{-1 / 2}(k+0.5-n p), \\
g(x, y, \varrho)=(2 \pi)^{-1}\left(1-Q^{2}\right)^{-1 / 2} \exp \left\{-\left(x^{2}+y^{2}-2 \varrho x y\right) / 2\left(1-e^{2}\right)\right\},
\end{gathered}
$$

and $\varrho$ is defined in (3.1.6).

In order to compute the $P\left[\bar{X} \geqslant \mu_{0}, N_{L} \leqslant k\right]$ using the approximation $P_{a}$, we need to know the mean $\mu$ and the variance $\sigma^{2}$ of the distribution in question, the proportion defective $p$ as defined in (3.1.2) and the correlation coefficient $Q$ as defined in (3.1.6). The computation of the bivariate normal term is described in more detail in Appendix $\mathbf{A}$.

\subsection{Normal Distribution}

Assume that the sample comes from a normal distribution $N\left(\mu, \sigma^{2}\right)$.

The item defective probability from (3.1.2) is

$$
p=P[X \leqslant L]=\Phi\{(L-\mu) / \sigma\},
$$

where $\Phi\{(L-\mu) / \sigma\}$ is the cdf of the $N(0,1)$ given in $(1.5)$.

In order to compute the approximation $P_{a}$ given in (3.1.9), we need to compute the correlation coefficient given in (3.1.6).

The expectation $E\left\{(X-\mu) I_{[X \leqslant L]}\right\}$ is evaluated as

$$
E\left\{(\mathrm{X}-\mu) I_{[X \leqslant L]}\right\}=-\frac{\sigma}{\sqrt{2 \pi}} \exp \left\{-(L-\mu)^{2} / 2 \sigma^{2}\right\} .
$$

Consequently the correlation coefficient is

$$
\mathrm{Q}=-(2 \pi p(1-p))^{-1 / 2} \exp \left\{-(L-\mu)^{2} / 2 \sigma^{2}\right\}
$$

In order to compare the approximation $P_{t}$ in (3.1.9) with an approximation developed by Elder and Muse [8], the lower limit $L$ is chosen under the assumption that $\mu=0, \sigma=1$, and according to the criterion

$$
P\left[N_{L} \leqslant k\right]=1-\alpha,
$$

where $0<\alpha<1$.

Because $N_{L}$ is $B(n, p)$, the lower limit $L$ is determined from

where $p=\Phi(L)$.

$$
\sum_{j=0}^{k}\left(\begin{array}{l}
n \\
i
\end{array}\right) p^{i(1-p)^{n-j}=1-\alpha,}
$$

Values of $L$ as tabulated by Elder and Muse for $\alpha=0.10,0.05$, and 0.01 are shown in table I. Once 
TABLE I. Lower Limits used in Computation of Acceptance Probabilities for Normal Distribution

\begin{tabular}{|c|c|c|c|c|}
\hline \multirow[b]{2}{*}{$n$} & \multirow[b]{2}{*}{$k$} & \multicolumn{3}{|c|}{ Lower Limit $L$} \\
\hline & & $\alpha=0.10$ & $\alpha=0.05$ & $\alpha=0.01$ \\
\hline 5 & $\begin{array}{l}0 \\
1 \\
2\end{array}$ & $\begin{array}{l}2.036 \\
1.215 \\
0.685\end{array}$ & $\begin{array}{l}2.319 \\
1.429 \\
0.881\end{array}$ & $\begin{array}{l}2.877 \\
1.843 \\
1.250\end{array}$ \\
\hline 10 & $\begin{array}{l}0 \\
1 \\
2\end{array}$ & $\begin{array}{l}2.309 \\
1.602 \\
1.196\end{array}$ & $\begin{array}{l}2.568 \\
1.789 \\
1.358\end{array}$ & $\begin{array}{l}3.089 \\
2.157 \\
1.670\end{array}$ \\
\hline 20 & $\begin{array}{l}0 \\
1 \\
2\end{array}$ & $\begin{array}{l}2.559 \\
1.928 \\
1.586\end{array}$ & $\begin{array}{l}2.799 \\
2.095 \\
1.726\end{array}$ & $\begin{array}{l}3.289 \\
2.428 \\
2.001\end{array}$ \\
\hline 30 & $\begin{array}{l}0 \\
1 \\
2\end{array}$ & $\begin{array}{l}2.696 \\
2.100 \\
1.783\end{array}$ & $\begin{array}{l}2.928 \\
2.258 \\
1.914\end{array}$ & $\begin{array}{l}3.402 \\
2.574 \\
2.172\end{array}$ \\
\hline
\end{tabular}

$L$ is determined the correlation coefficient of $\bar{X}$ and $N_{L}$ can be evaluated as

$$
\varrho=-[2 \pi p(1-p)]^{-1 / 2} \exp \left\{-L^{2} / 2\right\} \text {. }
$$

The ${ }^{t}$ Elder-Muse approximation along with their exact results are compared with the corresponding values of $P_{a}$ in table II where $L$ is chosen such that $\alpha=0.10$.

The comparison with the exact values derived in [8] shows that even for small sample size $P_{a}$ provides an excellent approximation to the acceptance probability $P_{n}$, and its effectiveness increases as $k$ gets larger. When $k=0$, the percent error in $P_{a}$ as compared to the exact results is approximately 3 percent. For $k=1$, it is about 1 percent and for $k=2$, it is less than 1 percent. The percentage errors in both $P_{a}$ and the Elder-Muse approximation when $\mu=0$ are shown below.

\begin{tabular}{|c|c|c|c|c|c|c|}
\hline \multicolumn{7}{|c|}{ Percent Error in Approximations } \\
\hline & \multicolumn{2}{|c|}{$k=0$} & \multicolumn{2}{|c|}{$k=1$} & \multicolumn{2}{|c|}{$k=2$} \\
\hline$n$ & $P_{a}$ & $\begin{array}{l}\text { Elder } \\
\text { Muse }\end{array}$ & $P_{a}$ & $\begin{array}{l}\text { Elder } \\
\text { Muse }\end{array}$ & $P_{x}$ & $\begin{array}{l}\text { Elder } \\
\text { Muse }\end{array}$ \\
\hline 5 & 3.3 & 1.0 & 1.0 & 1.8 & 0.6 & 1.2 \\
\hline 10 & 3.1 & 0.6 & 1.0 & 1.0 & 0.6 & 1.2 \\
\hline 20 & 3.0 & 0.2 & 0.8 & 0.6 & 0.6 & 0.8 \\
\hline 30 & 2.6 & 0.2 & 0.8 & 0.8 & 0.4 & 0.6 \\
\hline
\end{tabular}

\subsection{Weibull Distribution}

Assume that the sample $X_{1}, \ldots, X_{n}$ comes from a two parameter Weibull distribution $W(\lambda, \theta)$ with scale parameter $\lambda$, shape parameter $\theta$ and $p d f$

$$
f(x)=(\theta / \lambda)(x / \lambda)^{\theta-1} \exp \left\{-(x / \lambda)^{\theta}\right\} \text { for } x>0, \lambda>0, \theta>0
$$

The mean and variance are

$$
\mu=\lambda \Gamma(1+1 / \theta)
$$

and

$$
o^{2}=\lambda^{2}\left\{\Gamma(1+2 / \theta)-[\Gamma(1+1 / \theta)]^{2}\right\}
$$


respectively where $\Gamma(\cdot)$ is the gamma function.

For $0<\theta \leqslant 1, X$ has a decreasing failure rate (DFR) distribution; for $\theta \geqslant 1, X$ has an increasing failure rate (IFR) distribution. For further information see Johnson and Kotz [13].

In the case of the Weibull distribution, the proportion defective $p$ is defined from (3.1.2) and (3.2.2) as

$$
p=[X \leqslant L]=1-\exp \left\{-(L / \lambda)^{\theta}\right\} .
$$

The expectation

$$
\begin{aligned}
E X I_{[X \leqslant L]} & =\frac{\theta}{\lambda} \int_{0}^{L} x(x / \lambda)^{\theta-1} \exp \left\{-(x / \lambda)^{\theta}\right\} d x \\
& =\lambda \mathrm{I}\left\{(L / \lambda)^{\theta}, 1 / \theta\right\}
\end{aligned}
$$

and $I(c, d)$ is related to the incomplete $\Gamma$-function [12].

Combining (3.1.6), (3.3.4) and (3.3.5), we find that the correlation coefficient is

Table Il. Comparison of Approximation $\mathrm{P}_{\mathrm{a}}$ with

$$
\text { Elder-Muse Values for }
$$

\begin{tabular}{|c|c|c|c|c|c|c|c|c|c|c|}
\hline \multirow[b]{2}{*}{$n$} & \multicolumn{4}{|c|}{$k=0$} & \multicolumn{3}{|c|}{$k=1$} & \multicolumn{3}{|c|}{$k=2$} \\
\hline & $\mu$ & Exact & $P_{a}$ & $\begin{array}{l}\text { Elder } \\
\text { Muse }\end{array}$ & Exact & $P_{a}$ & $\begin{array}{l}\text { Elder } \\
\text { Muse }\end{array}$ & Exact & $P_{a}$ & $\begin{array}{l}\text { Elder } \\
\text { Muse }\end{array}$ \\
\hline \multirow[t]{9}{*}{5} & -.8 & 0.035 & 0.034 & 0.032 & 0.036 & 0.036 & 0.034 & 0.037 & 0.036 & 0.037 \\
\hline & -.6 & 0.087 & 0.082 & 0.085 & 0.089 & 0.088 & 0.089 & 0.089 & 0.089 & 0.091 \\
\hline & -.4 & 0.180 & 0.168 & 0.181 & 0.184 & 0.181 & 0.188 & 0.185 & 0.184 & 0.189 \\
\hline & -.2 & 0.318 & 0.300 & 0.323 & 0.324 & 0.320 & 0.332 & 0.326 & 0.325 & 0.333 \\
\hline & .0 & 0.488 & 0.472 & 0.493 & 0.496 & $0.49 \mathrm{~J}$ & 0.505 & 0.499 & 0.496 & 0.505 \\
\hline & .2 & 0.659 & 0.667 & 0.663 & 0.669 & 0.672 & 0.674 & 0.671 & 0.672 & 0.674 \\
\hline & .4 & 0.801 & 0.814 & 0.802 & 0.811 & 0.814 & 0.811 & 0.813 & 0.814 & 0.812 \\
\hline & .0 & 0.899 & 0.910 & 0.899 & 0.908 & 0.910 & 0.906 & 0.909 & 0.910 & 0.906 \\
\hline & .8 & 0.956 & 0.963 & 0.955 & 0.962 & 0.963 & 0.959 & 0.963 & 0.963 & 0.959 \\
\hline \multirow[t]{8}{*}{10} & -.6 & 0.027 & 0.026 & 0.026 & 0.028 & 0.028 & 0.026 & 0.028 & 0.028 & 0.027 \\
\hline & -.4 & 0.098 & 0.091 & 0.097 & 0.100 & 0.098 & 0.099 & 0.101 & 0.101 & 0.101 \\
\hline & -.2 & 0.252 & 0.236 & 0.253 & 0.257 & 0.252 & 0.261 & 0.260 & 0.257 & 0.264 \\
\hline & .0 & 0.480 & 0.465 & 0.483 & 0.490 & 0.485 & 0.495 & 0.494 & 0.491 & 0.500 \\
\hline & .2 & 0.713 & 0.732 & 0.714 & 0.725 & 0.735 & 0.728 & 0.731 & 0.736 & 0.733 \\
\hline & .4 & 0.876 & 0.897 & 0.876 & 0.888 & 0.897 & 0.887 & 0.893 & 0.897 & 0.891 \\
\hline & .0 & 0.956 & 0.971 & 0.956 & 0.966 & 0.971 & 0.965 & 0.969 & 0.971 & 0.967 \\
\hline & .8 & 0.956 & 0.994 & 0.985 & 0.992 & 0.994 & 0.991 & 0.993 & 0.994 & 0.993 \\
\hline \multirow[t]{6}{*}{20} & -.4 & 0.034 & 0.032 & 0.034 & 0.035 & 0.034 & 0.034 & 0.036 & 0.035 & 0.034 \\
\hline & -.2 & 0.174 & 0.162 & 0.174 & 0.178 & 0.173 & 0.178 & 0.180 & 0.177 & 0.181 \\
\hline & .0 & 0.474 & 0.460 & 0.475 & 0.483 & 0.479 & 0.486 & 0.488 & 0.485 & 0.492 \\
\hline & .2 & 0.781 & 0.811 & 0.781 & 0.795 & 0.814 & 0.795 & 0.802 & 0.814 & 0.802 \\
\hline & .4 & 0.937 & 0.963 & 0.937 & 0.950 & 0.963 & 0.950 & 0.956 & 0.963 & 0.955 \\
\hline & .6 & 0.981 & 0.996 & 0.981 & 0.991 & 0.996 & 0.991 & 0.994 & 0.996 & 0.993 \\
\hline \multirow[t]{6}{*}{30} & -.4 & 0.013 & 0.012 & 0.013 & 0.013 & 0.013 & 0.013 & 0.014 & 0.014 & 0.013 \\
\hline & -.2 & 0.127 & 0.118 & 0.127 & 0.130 & 0.126 & 0.129 & 0.131 & 0.129 & 0.131 \\
\hline & .0 & 0.470 & 0.458 & 0.471 & 0.479 & 0.476 & 0.480 & 0.484 & 0.482 & 0.487 \\
\hline & .2 & 0.824 & 0.861 & 0.824 & 0.839 & 0.863 & 0.839 & 0.847 & 0.863 & 0.846 \\
\hline & .4 & 0.958 & 0.986 & 0.958 & 0.972 & 0.986 & 0.972 & 0.978 & 0.986 & 0.977 \\
\hline & .6 & 0.985 & 0.999 & 0.985 & 0.995 & 0.999 & 0.995 & 0.997 & 0.999 & 0.997 \\
\hline
\end{tabular}

$P\left[X \geqslant \mu_{0}{ }^{*}, N_{l} \leqslant k\right]$ where $P\left(N_{l} \leqslant k\right)=0.90$

for Normal Distribution $N(0,1)$

$*_{0}=0$ 


$$
\varrho=\left[\lambda I\left\{(L / \lambda)^{\theta}, 1 / \theta\right\}-\mu p\right] / \sigma(p(1-p)\}^{1 / 2}
$$

where $\mu$ and $\sigma$ are defined by (3.3.2), and (3.3.3) respectively.

The limits of integration for the approximation (3.1.9) are

$$
\mathbf{a}=\frac{\mathbf{n}^{1 / 2}\left[\mu_{0}-\lambda \Gamma(1+1 / \theta)\right]}{\lambda\left\{\Gamma(1+2 / \theta)-[\Gamma(1+1 / \theta)]^{2}\right\}^{1 / 2}}
$$

and $b$ as defined in (3.1.11).

As is the case in the normal distribution, the lower limit $L$ is determined according to $(3.2 .3)$ and (3.3.4) for specified values of $k$ and $\alpha$.

Explicitly

$$
L=\lambda\left[-\log _{\mathrm{e}}(1-p)\right]^{1 / \theta} \text {. }
$$

The proportion defective $\mathrm{p}$ is tabulated in table III for $\alpha=0.10,0.05$ and $0.01, n=5,10,20,30$ and $k=0,1,2,3$. Corresponding lower limits $L$ where $\lambda=1$ are shown in table IV.

TABLE III. Proportion Defectives pused in Computation of Acceptance Probabilities

\begin{tabular}{l|c|c|c|c}
\hline \hline & & \multicolumn{3}{|c}{ Proportion Defective $p$} \\
\hline$n$ & $k$ & $\alpha=0.10$ & $\alpha=0.05$ & $\alpha=0.01$ \\
\hline$j$ & 0 & 0.0208 & 0.0102 & 0.00200 \\
& 1 & .112 & .0765 & .0330 \\
& 2 & .247 & .1890 & .106 \\
& 3 & .416 & .3425 & .222 \\
10 & 0 & 0.0105 & 0.00511 & 0.00100 \\
& 1 & .0545 & .0365 & .0155 \\
& 2 & .1153 & .0870 & .0475 \\
& 3 & .3875 & .1500 & .0930 \\
20 & 0 & 0.00525 & & 0.000500 \\
& 1 & .0269 & 0.00256 & .00759 \\
& 2 & .0564 & .0180 & .0227 \\
& 3 & .0902 & .0422 & .0435 \\
30 & 0 & 0.00350 & .0713 & \\
& 1 & .0178 & 0.00171 & .000335 \\
& 2 & .0373 & .0120 & .00500 \\
& 3 & .0594 & .0278 & .0285 \\
\hline
\end{tabular}

The approximation $P_{a}$ is compared to a simulation study where the acceptance probability was computed from 5,000 random samples. Simulation for the Weibull distribution was done by generating independent uniform random deviates $U_{i}$ using a congruential random number generator and making the transformation

$$
X_{i}=\lambda\left(-\log _{e} U_{i}\right)^{1 / \theta}
$$

The $X_{i}$ are independent $W(\lambda, \theta)$ r.v.s with pdf as shown in (3.3.1).

Values of $P_{a}$ and simulated acceptance probabilities are tabulated in table $V$ for $W$ eibull distribution $W(1, \theta)$ for $\theta=1,2,3.5$. 
The accuracy of the approximation $P_{a}$ as gauged by the simulation results is dependent on several factors; i.e., namely, the value of the shape parameter $\theta ; \alpha$. the probability that the sample will contain more than the allowable number of defectives; $n$, the size of the sample; and $k$, the number of allowable defectives or number of measurements less than the lower limit $L$.

The worst accuracy is for a Weibull distribution with $\theta=1$ where $\alpha$ is small, $\alpha=0.01$, and $n$ is small, $n=5$. The error is 9 percent for this case but drops to 2 percent when the sample size is in-

TABLE IV. Lower Limits Used in Compucation of Acceptance Probabilities for F́' eibull Distribution

\begin{tabular}{|c|c|c|c|c|c|}
\hline & & & & wer Limit & \\
\hline & $n$ & $k$ & $a=0.10$ & $a=0.05$ & $a=0.01$ \\
\hline$\theta=1$ & $\begin{array}{r}5 \\
5 \\
5 \\
5 \\
10 \\
10 \\
10 \\
10 \\
20 \\
20 \\
20 \\
20 \\
30 \\
30 \\
30 \\
30\end{array}$ & $\begin{array}{l}0 \\
1 \\
2 \\
3 \\
0 \\
1 \\
2 \\
3 \\
0 \\
1 \\
2 \\
3 \\
0 \\
1 \\
2 \\
3\end{array}$ & $\begin{array}{r}0.0210 \\
.1188 \\
.2837 \\
.5379 \\
.0106 \\
.0560 \\
.1227 \\
.2076 \\
.0053 \\
.0273 \\
.0581 \\
.0945 \\
.0035 \\
.0180 \\
.0380 \\
.0612\end{array}$ & $\begin{array}{r}0.0103 \\
.0790 \\
.2095 \\
.4193 \\
.0051 \\
.0372 \\
.0910 \\
.1025 \\
.0020 \\
.0189 \\
.0431 \\
.0740 \\
.0017 \\
.0121 \\
.0282 \\
.0479\end{array}$ & $\begin{array}{r}0.0020 \\
.0336 \\
.1120 \\
.2510 \\
.0010 \\
.0150 \\
.0487 \\
.0970 \\
.0005 \\
.0076 \\
.0230 \\
.0445 \\
.0003 \\
.0050 \\
.0150 \\
.0289\end{array}$ \\
\hline$\theta=2$ & $\begin{array}{r}5 \\
5 \\
5 \\
5 \\
10 \\
10 \\
10 \\
10 \\
20 \\
20 \\
20 \\
20 \\
30 \\
30 \\
30 \\
30\end{array}$ & $\begin{array}{l}0 \\
1 \\
2 \\
3 \\
0 \\
1 \\
2 \\
3 \\
0 \\
1 \\
2 \\
3 \\
0 \\
1 \\
2 \\
3\end{array}$ & $\begin{array}{l}.1450 \\
.3446 \\
.5326 \\
.7334 \\
.1027 \\
.2367 \\
.3503 \\
.4557 \\
.0726 \\
.1651 \\
.2409 \\
.3075 \\
.0592 \\
.1340 \\
.1950 \\
.2475\end{array}$ & $\begin{array}{l}.1013 \\
.2821 \\
.4575 \\
.6475 \\
.0716 \\
.1928 \\
.3017 \\
.4031 \\
.0506 \\
.1348 \\
.2076 \\
.2720 \\
.0414 \\
.1099 \\
.1679 \\
.2189\end{array}$ & $\begin{array}{l}.044 i \\
.1832 \\
.334 \bar{i} \\
.5010 \\
.0316 \\
.1250 \\
.2206 \\
.3124 \\
.0224 \\
.0873 \\
.1515 \\
.2109 \\
.0183 \\
.0708 \\
.1225 \\
.1700\end{array}$ \\
\hline$\theta=3.5$ & $\begin{array}{r}5 \\
5 \\
5 \\
5 \\
10 \\
10 \\
10 \\
10 \\
20 \\
20 \\
20 \\
20 \\
30 \\
30 \\
30 \\
30\end{array}$ & $\begin{array}{l}0 \\
1 \\
2 \\
3 \\
0 \\
1 \\
2 \\
3 \\
0 \\
1 \\
2 \\
3 \\
0 \\
1 \\
2 \\
3\end{array}$ & $\begin{array}{l}.3317 \\
.5441 \\
.6977 \\
.8376 \\
.2724 \\
.4390 \\
.5492 \\
.6382 \\
.2233 \\
.3573 \\
.4434 \\
.5097 \\
.1988 \\
.3171 \\
.3929 \\
.4502\end{array}$ & $\begin{array}{l}.2702 \\
.4852 \\
.0398 \\
.7801 \\
.2210 \\
.3904 \\
.5042 \\
.5950 \\
.1818 \\
.3181 \\
.4073 \\
.4752 \\
.1620 \\
.2831 \\
.3607 \\
.4198\end{array}$ & $\begin{array}{l}.1694 \\
.3791 \\
.5351 \\
.6737 \\
.1390 \\
.3047 \\
.4216 \\
.5144 \\
.1140 \\
.2482 \\
.3402 \\
.4109 \\
.1017 \\
.2202 \\
.3013 \\
.3633\end{array}$ \\
\hline
\end{tabular}


TABLE V. Comparison of Approximation $P$ with Simulation for

$P\left(\bar{X} \geqslant \mu_{0}{ }^{*} . \mathrm{N}_{\mathrm{L}} \leqslant k\right)$ where $P\left(N_{L} \leqslant k\right)=1-a$

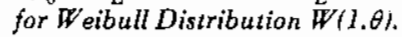

\begin{tabular}{|c|c|c|c|c|c|c|c|c|}
\hline & \multirow[b]{3}{*}{$\mathrm{n}$} & \multirow[b]{3}{*}{$\mathbf{k}$} & \multicolumn{6}{|c|}{ Probability of Acceptance } \\
\hline & & & \multicolumn{2}{|c|}{$\alpha=0.10$} & \multicolumn{2}{|c|}{$\alpha=0.05$} & \multicolumn{2}{|c|}{$\alpha=0.01$} \\
\hline & & & $P_{a}$ & Simul & $P_{a}$ & Simul & $P_{a}$ & Simul \\
\hline \multirow[t]{16}{*}{$\theta=1$} & 5 & 0 & 0.645 & 0.634 & 0.698 & 0.663 & 0.712 & 0.672 \\
\hline & $\tilde{s}$ & 1 & .668 & .652 & .698 & .673 & .712 & .672 \\
\hline & 5 & 2 & .678 & .664 & .699 & .680 & .711 & .674 \\
\hline & 5 & 3 & .688 & .674 & .702 & .684 & .711 & .675 \\
\hline & 10 & 0 & .705 & .707 & .768 & .755 & .785 & .773 \\
\hline & 10 & 1 & .726 & .724 & .768 & .763 & $.78 \tilde{5}$ & .776 \\
\hline & 10 & 2 & .733 & .734 & .766 & .768 & .785 & .778 \\
\hline & 10 & 3 & .738 & .743 & .767 & .772 & .784 & .779 \\
\hline & 20 & 0 & .775 & .795 & .848 & .828 & .868 & .875 \\
\hline & 20 & 1 & .795 & .801 & .846 & .836 & .868 & .877 \\
\hline & 20 & 2 & .798 & .807 & .844 & .840 & .867 & .879 \\
\hline & 20 & 3 & .801 & .810 & .843 & .841 & .867 & .878 \\
\hline & 30 & 0 & .815 & .826 & .893 & .872 & .914 & .914 \\
\hline & 30 & 1 & .835 & .837 & .890 & .879 & .914 & .918 \\
\hline & 30 & 2 & .837 & .838 & .887 & .878 & .914 & כ \\
\hline & 30 & 3 & .838 & .841 & .886 & .881 & .913 & .917 \\
\hline \multirow[t]{16}{*}{$\theta=2$} & 5 & 0 & .681 & .703 & .720 & .731 & .744 & .738 \\
\hline & 5 & 1 & .709 & .723 & .729 & .734 & .744 & .739 \\
\hline & 5 & 2 & .721 & .736 & .733 & .737 & .744 & .740 \\
\hline & 5 & 3 & .729 & .740 & .736 & .739 & .744 & .740 \\
\hline & 10 & 0 & .743 & .764 & .788 & .807 & .824 & .822 \\
\hline & 10 & 1 & .768 & .780 & .803 & .808 & .824 & .825 \\
\hline & 10 & 2 & .777 & .794 & .808 & .808 & .823 & .826 \\
\hline & 10 & 3 & .784 & .800 & .812 & .810 & .823 & .827 \\
\hline & 20 & 0 & .810 & .827 & .865 & .885 & .906 & .903 \\
\hline & 20 & l & .832 & .839 & .876 & .884 & .906 & .905 \\
\hline & 20 & 2 & .837 & .848 & .884 & .882 & .905 & .907 \\
\hline & 20 & 3 & .840 & .851 & .884 & .882 & .905 & .907 \\
\hline & 30 & 0 & .844 & .860 & .897 & .924 & .946 & .947 \\
\hline & 30 & 1 & .865 & .866 & .903 & .922 & .946 & .950 \\
\hline & 30 & 2 & .867 & .867 & .909 & .919 & .946 & .949 \\
\hline & 30 & 3 & .869 & .872 & .914 & .918 & .945 & .952 \\
\hline \multirow[t]{16}{*}{$\theta=3.5$} & 5 & 0 & .801 & .829 & .864 & .859 & .880 & .872 \\
\hline & 5 & 1 & .830 & .844 & .865 & .873 & .880 & .875 \\
\hline & 5 & 2 & .839 & .855 & .868 & .875 & .880 & .876 \\
\hline & 5 & 3 & .843 & .853 & .869 & .878 & .879 & .877 \\
\hline & 10 & 0 & .853 & .864 & .931 & .911 & .951 & .942 \\
\hline & 10 & 1 & .877 & .868 & .930 & .915 & .951 & .947 \\
\hline & 10 & 2 & .882 & .884 & .928 & .922 & .951 & .946 \\
\hline & 10 & 3 & .885 & .889 & .928 & .925 & .951 & .950 \\
\hline & 20 & 0 & .882 & .890 & .968 & .938 & .990 & .983 \\
\hline & 20 & 1 & .902 & .894 & .964 & .946 & .990 & .984 \\
\hline & 20 & 2 & .903 & .899 & .960 & .946 & .990 & .987 \\
\hline & 20 & 3 & .903 & .894 & .958 & .947 & .989 & .986 \\
\hline & 30 & 0 & .887 & .893 & .974 & .938 & .998 & .984 \\
\hline & 30 & 1 & .908 & .896 & .970 & .947 & .998 & .989 \\
\hline & 30 & 2 & .907 & .894 & .966 & .948 & .997 & .990 \\
\hline & 30 & 3 & .907 & .898 & .964 & .956 & .996 & .990 \\
\hline
\end{tabular}

${ }^{*} \mu_{0}=0.75$ 
creased to $n=10$. For other Weibull distributions and combinations of $\alpha$ and $n$, the worst accuracies occur when $k=0$, and in this case the errors are as large as 6 percent for $n=5$ and 4 percent for $n=$ 30. However, the approximation $P_{a}$ works very well when $k>0$. The disagreement between $P_{a}$ and the simulation is less than 1 percent for a large proportion of the points when $k>0$.

\subsection{Exponential Distribution}

Assume that the sample $X_{1}, \ldots, X_{n}$ comes from an exponential distribution $E(\lambda, \beta)$ with location parameter $\beta$ and scale parameter $\lambda$ and pdf

$$
f(x)=(1 / \lambda) \exp \{-(x-\beta) / \lambda\} \quad x>\beta, \lambda>0
$$

The mean and variance of $X$ are given by $\mu=\lambda+\beta$ and $\sigma^{2}=\lambda^{2}$ respectively.

We have

$$
p=1-\exp (-(L-\beta) / \lambda)
$$

and

$$
E X I_{[\mathrm{X} \leqslant \mathrm{L}]}=\lambda p-(1-p)(L-\beta)+\beta p .
$$

Combining (3.4.2) and (3.4.3), we get

$$
\varrho=-(1-p)^{1 / 2(L-\beta) / \lambda p^{1 / 2}}
$$

Using values for the proportion defective $p$ that are given in table III, the corresponding limits $L$ as determined by

$$
L=\beta-\lambda \log (1-p)
$$

are found in table VI for $\beta=0$ and $\lambda=0.5,1,2$.

The values $a$ and $b$ appearing in the approximation $P_{a}(3.1 .9)$ are given by

$$
\begin{aligned}
& a=n^{1 / 2} \lambda^{-1}\left(\mu_{0}-\lambda-\beta\right) \\
& b=(n p(1-p))^{-1 / 2}(k+0.5-n p)
\end{aligned}
$$

and $\varrho$ is defined by (3.4.4.)

Values of $P_{a}$ and simulated acceptance probabilities are tabulated in table VII for the exponential distribution $E(\lambda, 0)$ for $\lambda=0.5,1,2$.

The accuracy of the approximation $P_{a}$ is more dependent on $n$, the sample size and less dependent on $k$, the number of allowable defectives for the exponential distribution than for Weibull distributions. The worst accuracy is for an exponential distribution with $\lambda=1$, where $k=0$ and $n=5$. The disagreement with the simulation in this case is 7 percent, dropping to 1 percent when the sample size is increased to $n=10$. In general, the accuracies are not dependent upon the parameter $\lambda$ but are somewhat dependent upon the way in which the lower limit $L$ is chosen, and the accuracies tend to worsen as the probability of the sample containing more than the allowable number of defectives in. creases. Accuracies of about 2 percent are characteristic of the results over all values of $k$.

\section{A Lower Bound for the Acceptance Probability}

A lower bound for the acceptance probability is provided by the following lemma.

LEMMA 4.1: Let $\mathrm{X}_{1}, \ldots, \mathrm{X}_{\mathrm{n}}$ be i.i.d random variables from a continuous distribution. Let $\overline{\mathrm{X}}$ be the 
sample mean and $\mathrm{X}_{(r)}$ be the $r^{\text {th }}$ smallest order statistic of $\mathrm{X}_{1}, \ldots, \mathrm{X}_{\mathrm{n}}$. Then for arbitrarily fixed real numbers $\mathrm{a}, \mathrm{b}$ and positive integer $r, 1 \leqslant r \leqslant \mathrm{n}$,

$$
\begin{aligned}
& \mathrm{P}\left[\overline{\mathrm{X}} \geqslant \mathrm{a}, \mathrm{X}_{(r)} \geqslant \mathrm{b}\right] \geqslant \mathrm{P}[\overline{\mathrm{X}} \geqslant \mathrm{a}] \mathrm{P}\left[\mathrm{X}_{(r)} \geqslant \mathrm{b}\right] \\
& \mathrm{P}\left[\overline{\mathrm{X}}<\mathrm{a}, \mathrm{X}_{(r)}<\mathrm{b}\right] \geqslant \mathrm{P}[\overline{\mathrm{X}}<\mathrm{a}] \mathrm{P}\left[\mathrm{X}_{(r)}<\mathrm{b}\right] .
\end{aligned}
$$

The lemma is an easy eonsequenee of a general theorem (Esary, Proschan, and Walkup [10]). For easy reference, we quote the theorem below, as well as the definition of "associatedness." Random

\begin{tabular}{|c|c|c|c|c|c|}
\hline & & & \multicolumn{3}{|c|}{ Lower Limit L } \\
\hline & $\mathbf{n}$ & $\mathbf{k}$ & $\alpha=0.10$ & $\alpha=0.05$ & $\alpha=0.01$ \\
\hline$\lambda=0.5$ & $\begin{array}{r}5 \\
5 \\
5 \\
5 \\
10 \\
10 \\
10 \\
10 \\
20 \\
20 \\
20 \\
20 \\
30 \\
30 \\
30 \\
30\end{array}$ & $\begin{array}{l}0 \\
1 \\
2 \\
3 \\
0 \\
1 \\
2 \\
3 \\
0 \\
1 \\
2 \\
3 \\
0 \\
1 \\
2 \\
3\end{array}$ & $\begin{array}{r}0.0105 \\
.0594 \\
.1418 \\
.2689 \\
.0053 \\
.0280 \\
.0614 \\
.1038 \\
.0026 \\
.0136 \\
.0290 \\
.0473 \\
.0018 \\
.0090 \\
.0190 \\
.0306\end{array}$ & $\begin{array}{r}0.0051 \\
.0398 \\
.1047 \\
.2097 \\
.0026 \\
.0186 \\
.0455 \\
.0813 \\
.0013 \\
.0091 \\
.0216 \\
.0370 \\
.0009 \\
.0060 \\
.0141 \\
.0240\end{array}$ & $\begin{array}{r}0.0010 \\
.0168 \\
.0560 \\
.1255 \\
.0005 \\
.0078 \\
.0243 \\
.0488 \\
.0003 \\
.0038 \\
.0115 \\
.0222 \\
.0002 \\
.0025 \\
.0075 \\
.0145\end{array}$ \\
\hline$\lambda=1.0$ & $\begin{array}{r}5 \\
5 \\
5 \\
5 \\
10 \\
10 \\
10 \\
10 \\
20 \\
20 \\
20 \\
20 \\
30 \\
30 \\
30 \\
30\end{array}$ & $\begin{array}{l}0 \\
1 \\
2 \\
3 \\
0 \\
1 \\
2 \\
3 \\
0 \\
1 \\
2 \\
3 \\
0 \\
1 \\
2 \\
3\end{array}$ & $\begin{array}{l}.0210 \\
.1188 \\
.2837 \\
.5379 \\
.0106 \\
.0560 \\
.1227 \\
.2076 \\
.0053 \\
.0273 \\
.0581 \\
.0945 \\
.0035 \\
.0180 \\
.0380 \\
.0612\end{array}$ & $\begin{array}{l}.0103 \\
.0796 \\
.2095 \\
.4193 \\
.0051 \\
.0372 \\
.0910 \\
.1625 \\
.0026 \\
.0182 \\
.0431 \\
.0740 \\
.0017 \\
.0121 \\
.0282 \\
.0479\end{array}$ & $\begin{array}{l}.0020 \\
.0336 \\
.1120 \\
.2510 \\
.0010 \\
.0150 \\
.0487 \\
.0976 \\
.0005 \\
.0076 \\
.0230 \\
.0445 \\
.0003 \\
.0050 \\
.0150 \\
.0289\end{array}$ \\
\hline$\lambda=2$ & $\begin{array}{r}5 \\
5 \\
5 \\
5 \\
10 \\
10 \\
10 \\
10 \\
20 \\
20 \\
20 \\
20 \\
30 \\
30 \\
30 \\
30\end{array}$ & $\begin{array}{l}0 \\
1 \\
2 \\
3 \\
0 \\
1 \\
2 \\
3 \\
0 \\
1 \\
2 \\
3 \\
0 \\
1 \\
2 \\
3\end{array}$ & $\begin{array}{r}.0420 \\
.2376 \\
.5674 \\
1.0757 \\
.0211 \\
.1121 \\
.2455 \\
.4153 \\
.0105 \\
.0545 \\
.1161 \\
.1891 \\
.0070 \\
.0359 \\
.0760 \\
.1225\end{array}$ & $\begin{array}{l}.0205 \\
.1592 \\
.4190 \\
.8386 \\
.0102 \\
.0744 \\
.1820 \\
.3250 \\
.0051 \\
.0363 \\
.0862 \\
.1479 \\
.0034 \\
.0241 \\
.0564 \\
.0959\end{array}$ & $\begin{array}{l}.0040 \\
.0671 \\
.2241 \\
.5021 \\
.0020 \\
.0312 \\
.0973 \\
.1952 \\
.0010 \\
.0152 \\
.0459 \\
.0889 \\
.0007 \\
.0100 \\
.0300 \\
.0578\end{array}$ \\
\hline
\end{tabular}

Table VI. Lower Limits used in Computation of Acceptance Probabilities for Exponential Distribution 


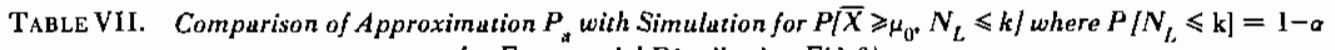
for Exponential Distribution E( $\left.\lambda_{4}, 0\right)$

\begin{tabular}{|c|c|c|c|c|c|c|c|}
\hline \multirow[b]{3}{*}{$\mathrm{n}$} & \multirow[b]{3}{*}{$\mathbf{k}$} & \multicolumn{6}{|c|}{ Probability of Acceptance } \\
\hline & & \multicolumn{2}{|c|}{$a=0.10$} & \multicolumn{2}{|c|}{$\alpha=0.05$} & \multicolumn{2}{|c|}{$\alpha=0.01$} \\
\hline & & $P_{a}$ & Simul & $P_{a}$ & Simul & $P_{a}$ & Simul \\
\hline \multicolumn{8}{|c|}{$\lambda=0.5, \mu_{0}=0.25$} \\
\hline 5 & 0 & 0.781 & 0.825 & 0.850 & 0.853 & 0.868 & 0.893 \\
\hline 5 & 1 & .804 & .846 & .847 & .871 & .868 & .896 \\
\hline 5 & 2 & .811 & .857 & .846 & .874 & .867 & .897 \\
\hline 5 & 3 & .819 & .869 & .847 & .885 & .866 & .899 \\
\hline 10 & 0 & .842 & .875 & .922 & .929 & .943 & .957 \\
\hline 10 & 1 & .862 & .889 & .919 & .927 & .943 & .958 \\
\hline 10 & 2 & .865 & .893 & .915 & .933 & .942 & .958 \\
\hline 10 & 3 & .867 & .897 & .913 & .935 & .941 & .956 \\
\hline 20 & 0 & .878 & .902 & .964 & .948 & .987 & .985 \\
\hline 20 & 1 & .898 & .905 & .960 & .954 & .987 & .984 \\
\hline 20 & 2 & .898 & .907 & .956 & .955 & .986 & .984 \\
\hline 20 & 3 & .898 & .899 & .953 & .948 & .985 & .984 \\
\hline 30 & 0 & .886 & .901 & .973 & .952 & .997 & .987 \\
\hline 30 & I & .906 & .904 & .969 & .951 & .997 & .990 \\
\hline 30 & 2 & .906 & .903 & .965 & .948 & .996 & .990 \\
\hline 30 & 3 & .906 & .903 & .962 & .950 & .995 & .989 \\
\hline \multicolumn{8}{|c|}{$\lambda=1.0, \mu_{0}=0.75$} \\
\hline 5 & 0 & .645 & .632 & .698 & .651 & .712 & .686 \\
\hline 5 & 1 & .668 & .657 & .698 & .660 & .712 & .689 \\
\hline 5 & 2 & .673 & .665 & .699 & .667 & .711 & .690 \\
\hline$\tilde{5}$ & 3 & .688 & .676 & .702 & .674 & .711 & .691 \\
\hline 10 & 0 & .705 & .712 & .768 & .754 & .785 & .776 \\
\hline 10 & 1 & .726 & .726 & .768 & $.76]$ & .785 & .777 \\
\hline 10 & 2 & .733 & .734 & .767 & .766 & .785 & .777 \\
\hline 10 & 3 & .738 & .745 & .767 & .769 & .784 & .778 \\
\hline 20 & 0 & .775 & .789 & .848 & .846 & .868 & .873 \\
\hline 20 & 1 & .795 & .800 & .846 & .849 & .868 & .876 \\
\hline 20 & 2 & .798 & .804 & .844 & .849 & .867 & .875 \\
\hline 20 & 3 & .801 & .806 & .843 & .851 & .867 & .873 \\
\hline 30 & 0 & .815 & .839 & .893 & .890 & .915 & .920 \\
\hline 30 & 1 & .835 & .846 & .890 & .887 & .914 & .921 \\
\hline 30 & 2 & .837 & .842 & .887 & .884 & .914 & .922 \\
\hline 30 & 3 & .838 & .846 & .886 & .891 & .913 & .921 \\
\hline \multicolumn{8}{|c|}{$\lambda=2.0, \mu_{0}=0.75$} \\
\hline 5 & 0 & .825 & .872 & .899 & .915 & .919 & .950 \\
\hline 5 & 1 & .846 & .883 & .895 & .927 & .919 & .954 \\
\hline 5 & 2 & .851 & .891 & .893 & .929 & .918 & .953 \\
\hline 5 & 3 & .856 & .894 & .892 & .935 & .916 & .955 \\
\hline 10 & 0 & .870 & .894 & .954 & .944 & .976 & .987 \\
\hline 10 & 1 & .889 & .889 & .950 & .944 & .976 & .987 \\
\hline 10 & 2 & .891 & .898 & .945 & .948 & .975 & .986 \\
\hline 10 & 3 & .891 & .894 & .942 & .945 & .974 & .985 \\
\hline 20 & 0 & .887 & .902 & .974 & .952 & .997 & .991 \\
\hline 20 & 1 & .906 & .903 & .970 & .956 & .997 & .989 \\
\hline 20 & 2 & .906 & .905 & .965 & .956 & .996 & .991 \\
\hline 20 & 3 & .906 & .904 & .962 & .955 & .995 & .992 \\
\hline 30 & 0 & .889 & .907 & .976 & .956 & 1.000 & .990 \\
\hline 30 & 1 & .909 & .906 & .972 & .949 & .999 & .991 \\
\hline 30 & 2 & .908 & .902 & .968 & .948 & .999 & .991 \\
\hline 30 & 3 & .908 & .901 & .965 & .952 & .998 & .990 \\
\hline
\end{tabular}


variables $X_{1}, \ldots, X_{n}$ are said to be associated if

$$
\operatorname{Cov}\left(f\left(T^{7}\right), g\left(T^{\prime}\right) \geqslant 0\right.
$$

for all non-decreasing functions $f$ and $g$ in each $X_{\mathrm{j}}$ for which $E f(T), E g(T), E f(T) g(T)$ exist and $T$ denotes $\left\{X_{1}, \ldots, X_{n}\right\}$.

THEOREM 4.1. Let $\mathrm{T}=\left\{\mathrm{X}_{1}, \ldots, \mathrm{X}_{\mathrm{n}}\right\}$ be associated, $\mathrm{S}_{\mathrm{i}}=\mathrm{f}_{\mathrm{i}}(\mathrm{T})$ and $\mathrm{f}_{\mathrm{i}}$ be nondecreasing for $\mathrm{i}=1, \ldots, \mathrm{k}$. Then

$$
\begin{aligned}
& \mathrm{P}\left[\mathrm{S}_{1} \leqslant s_{1}, \ldots, \mathrm{S}_{\mathrm{k}} \leqslant \mathrm{s}_{\mathrm{k}}\right] \geqslant \prod_{i=1}^{k} \mathrm{P}\left[\mathrm{S}_{\mathrm{i}} \leqslant s_{i}\right] \\
& \mathrm{P}\left[\mathrm{S}_{1}>\mathrm{s}_{1}, \ldots, \mathrm{S}_{\mathrm{k}}>\mathrm{s}_{\mathrm{k}}\right] \geqslant \prod_{i=1}^{k} \mathrm{P}\left[S_{i}>s_{i}\right]
\end{aligned}
$$

for all $\mathrm{s}_{1}, \ldots, \mathrm{s}_{\mathbf{k}}$.

PROOF OF LEMMA 4.1: In our case the $X_{i}$ 's are statistically independent and hence associated. Let $S_{1}=\bar{X}$ and $S_{2}=X_{(r)}$. Clearly, $S_{1}$ and $S_{2}$ are non-decreasing functions in each of the $X_{i}$ 's; hence (4.1) and $(4.2)$ hold. Moreover, $\operatorname{Cov}\left(S_{1}, S_{2}\right)=\operatorname{Cov}\left(\bar{X}, X_{(r)}\right) \geqslant 0$. This completes the proof.

From Lemma 4.1, we have a lower bound $\underline{P}$ to the acceptance probability

$$
\underline{P}=P[\bar{X} \geqslant \mathrm{a}] P\left[X_{(k+1)}>L\right] \leqslant P_{n}=P\left[\bar{X} \geqslant a, X_{(k+1)}>L\right],
$$

where $k+1$ corresponds to $r$.

The r.v. $X_{(k+1)}$ can be transformed to a r.v. $Z$ with Beta distribution with parameters $n-k$ and $k+1$. Thus

$$
P\left[X_{(k+1)}>L\right]=P\left[Z<1-F_{X}(L)\right]=\frac{\Gamma(n+1)}{\Gamma(k+1) \Gamma(n-k)}-\int_{0}^{1-p} z^{n-k-1}(1-z)^{k} d z .
$$

The lower bound $\underline{P}$ in (4.5) can be computed using the marginal distribution of the sample mean and the Beta distribution.

Because the computation of the lower bound $P$ is much easier than the computation of the acceptance probability $P_{n}$ it would be an immense simplification if the lower bound could serve as an approximation for $P_{n}$.

Therefore, it is of practical importance to determine the sample size $n$ and values of $k$ that are necessary in order that the lower bound be an acceptable approximation for $P_{n}$. In other words, it is of interest to know the smallest value of $\mathbf{n}$ and the range of $k$ values which makes the independence of $\bar{X}$ and $X_{(k+1)}$ acceptable.

\section{Comparison of the Exact Probability of Acceptance with the Approximation and the Lower Bound}

\subsection{Acceptance Probability Curves}

The acceptance probabilities computed using either simulation or numerical integration along with the corresponding lower bound $\underline{P}$ and the approximation $P_{a}$ are plotted as a function of one parameter 
of the distribution in question. This provides a comparison of the relative accuracy of $P_{a}$ to $P$ as a technique for approximating $P_{n}$. The curves are varied over $n$ and $k$ in order to examine the effect of sample size and number of allowable defectives $k$ on $P_{n}, P_{a}$ and $\underline{P}$.

\subsection{Normal Distribution}

Assuming that $X_{l}, \ldots, X_{n}$ are i.i.d. $N(\mu, 1)$, the acceptance probability

$$
P_{n}=P_{\mu}\left[\bar{X} \geqslant \mu_{0}, X_{(k+1)}>L\right]
$$

for $L$ chosen according to (3.2.3) and $\mu_{0}=0$ was computed using a technique for simulating random normal deviates due to Box and Muller [3]. The resulting acceptance probabilities as a function of $\mu$ are shown as the solid line in figures 1-4.

The corresponding lower bound $\underline{P}$ was computed from (4.5) and the approximation $\overline{\mathrm{P}}_{\mathrm{a}}$ was computed for (3.1.9).

The relationships among the probability of acceptance $P_{n}$, its approximation $P_{a}$, and its lower bound $\underline{P}$ as a function of sample size $n$ and allowable number of defectives $k$ is depieted in figures 1-4 for samples of size $n=10$ and $n=30$. The following convention is used for all figures; namely, $P_{n}$ is shown as a solid line; $P_{a}$ is shown as a heavy dashed line; and $P$ is shown as a lighter dotted line.

From figure 1 it is obvious that when $k=0$ and $n$ is small, $P_{a}$ is a better approximation to the acceptance probability than the lower bound as long as $\mu<0.25$. As $n$ increases the superiority of $P_{a}$ to $\underline{P}$ increases as $k$ is allowed to become larger. For example, when $k=3$ as in figure 4, the lower bound
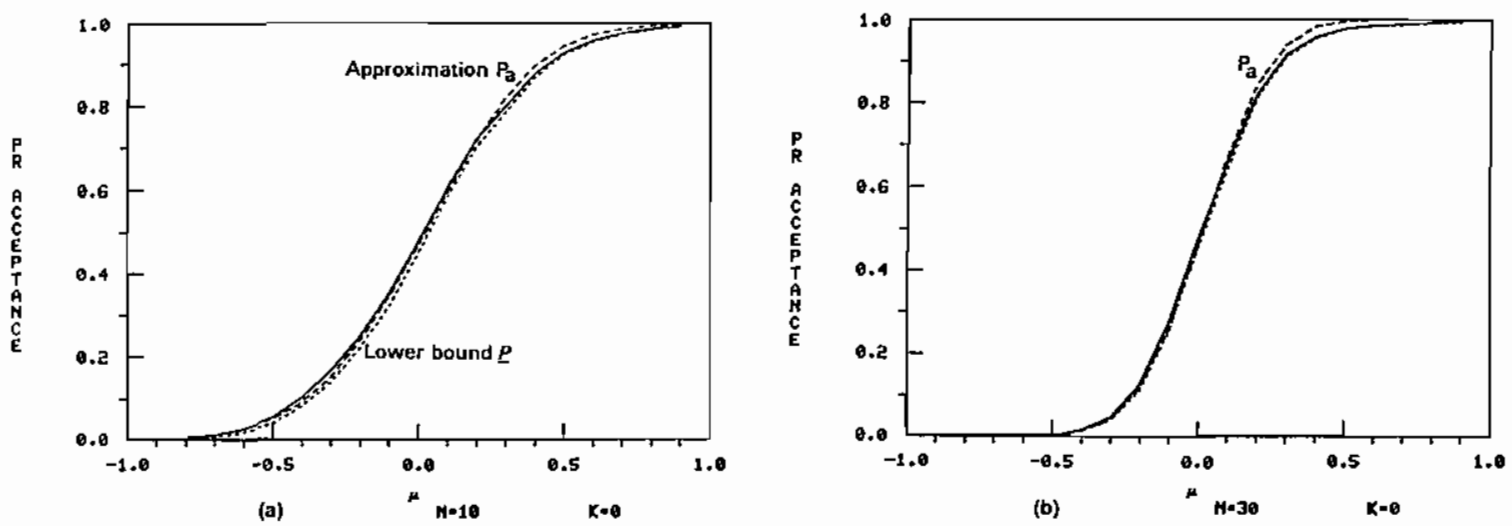

Figure 1. Acceptance probabilities when the number of allowable defeetives $k=0$ and $n$ observations are drawn from the normal distribution $N(\mu, 1)$.
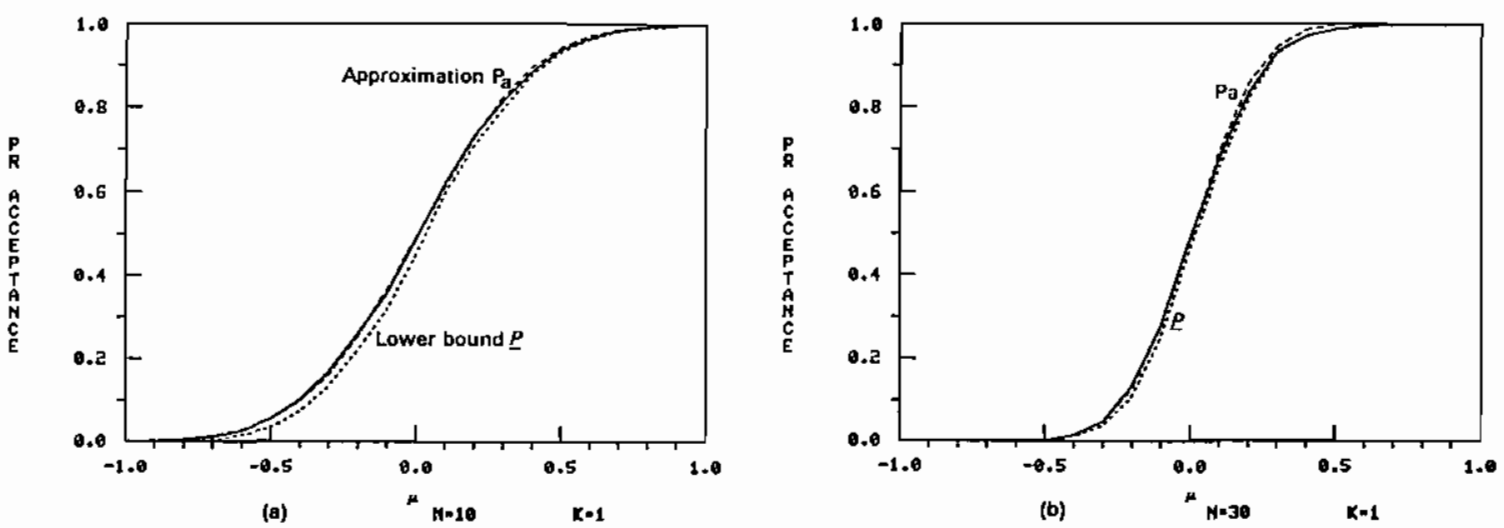

FIGURE 2. Acceptance probabilities when the number of allowable delectives $k=1$ and $n$ observations are drawn from the normal distribution $N(\mu, 1)$. 

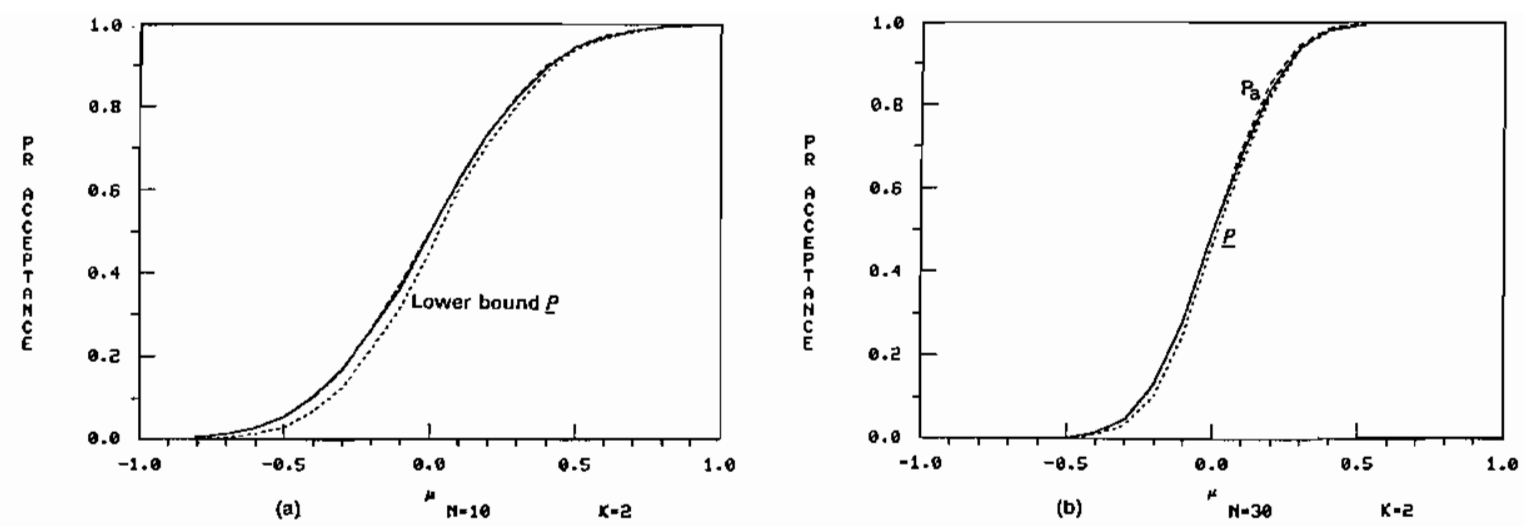

Figure 3. Acceptance probabilities when the number of allowable defectives $k=2$ and $n$ observations are drawn from the normal distributiou $N(\mu, 1)$.
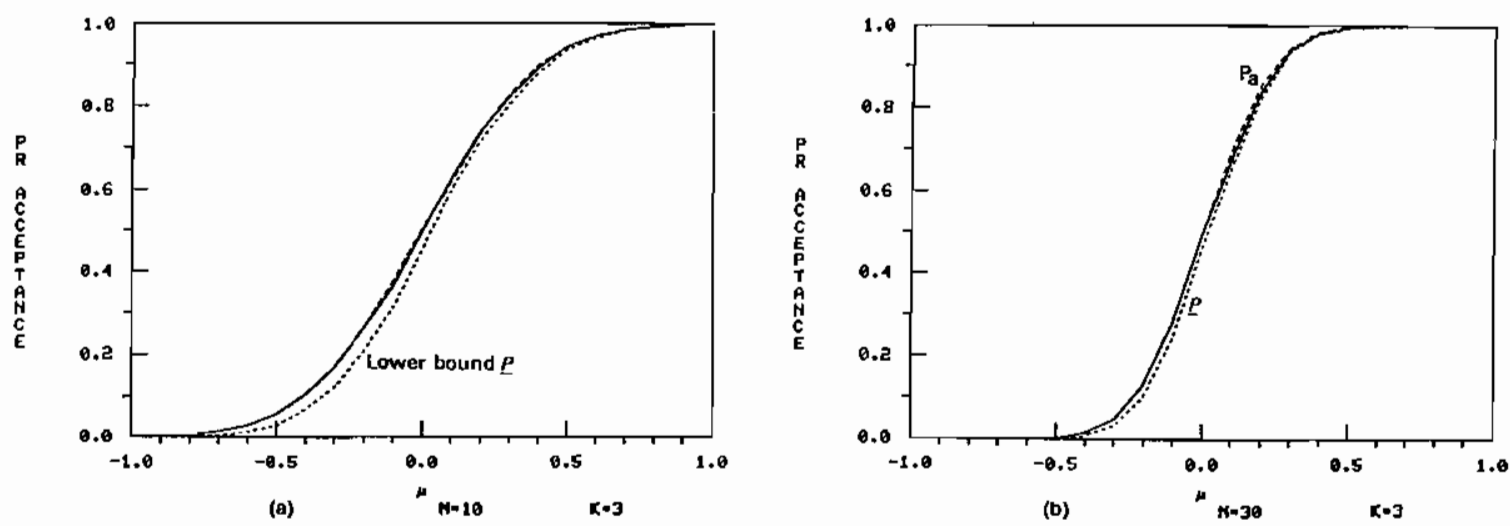

FigURE 4. Aceeptance probabilities when the number of allowable defectives $k=3$ and $n$ observations are drawn from the normal distribution $N(\mu, 1)$.

does not give a satisfactory approximation for the smaller sample size, and $\boldsymbol{P}_{a}$ is clearly preferable. Evcn for $n=30, P_{a}$ is at least as accurate as $\underline{P}$ over the entire range of $\mu$.

\subsection{Weibull Distribution}

Assuming that $X_{1}, \ldots, X_{n}$ are i.i.d. $W(1, \theta)$, and that $\mu_{0}=0.75$ and that $L$ is chosen according to (3.3.8) with $\theta=1$, the acceptance probability was computed by simulation and is shown as the solid line in figurcs 5-8. The corresponding lower bound $\underline{P}$ was also computed using simulation and is
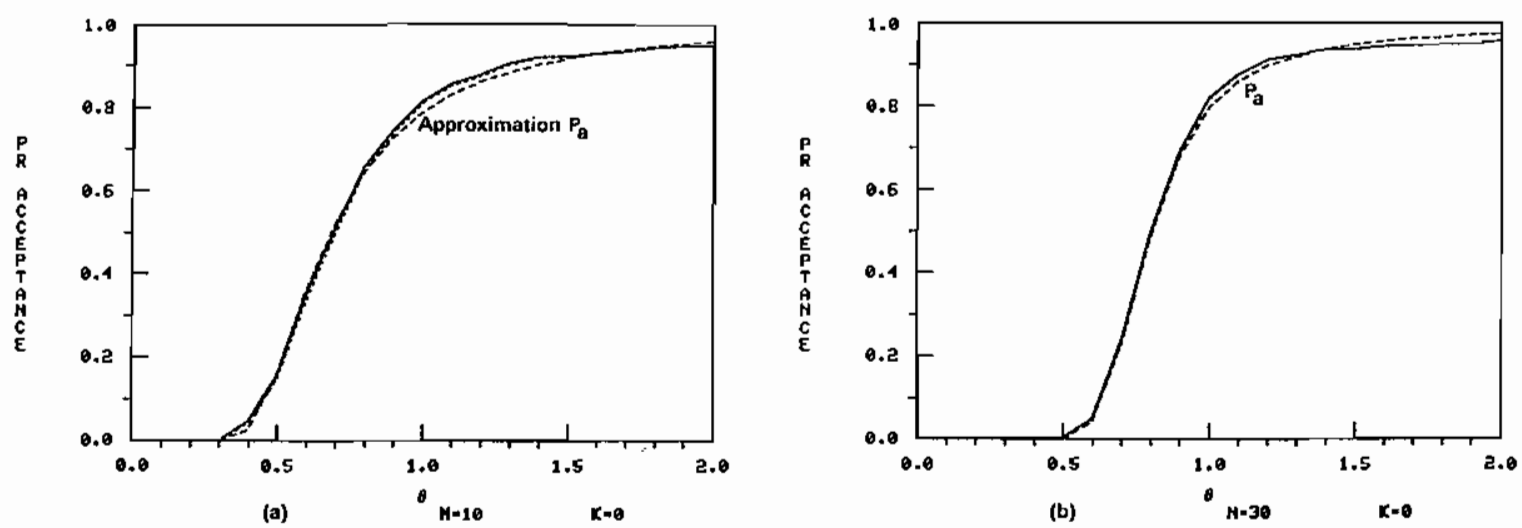

Figure 5. Acceptance probabilities when the number of allowable defectives $k=0$ and $n$ observations are drawn from a Weibull distribution $W(1, \theta)$. 

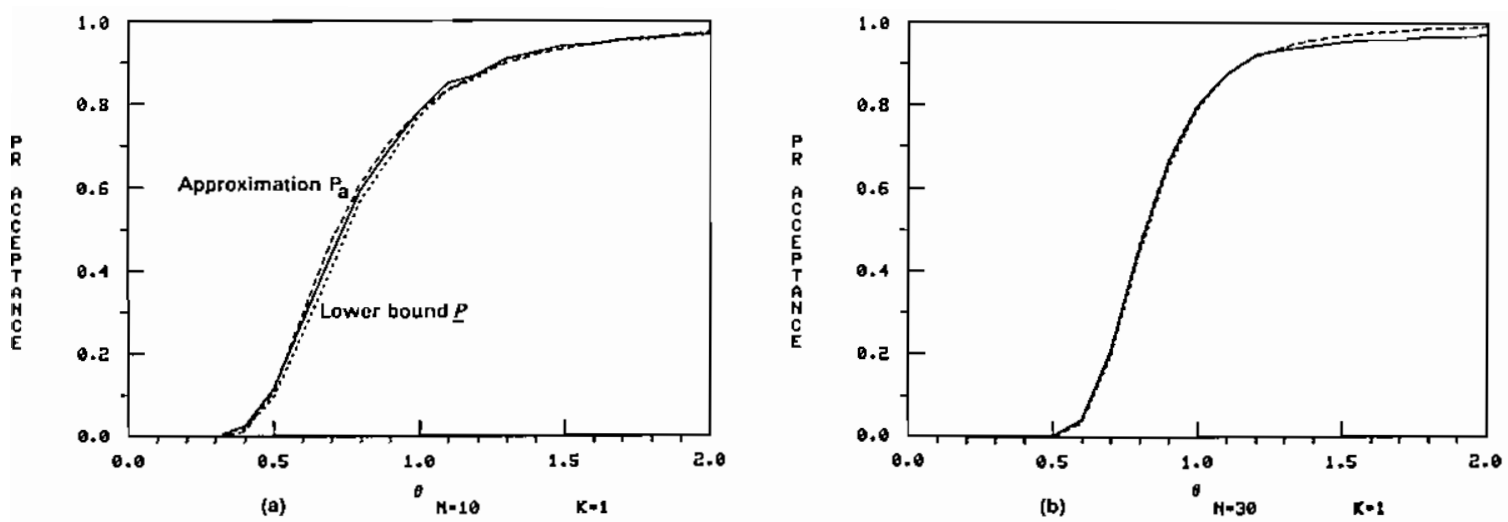

Figure 6. Acceptance probabilities when the number of allowable defectives $k=1$ and $n$ observations are drawn Jrom a Weibull distribution $W(1, \theta)$.
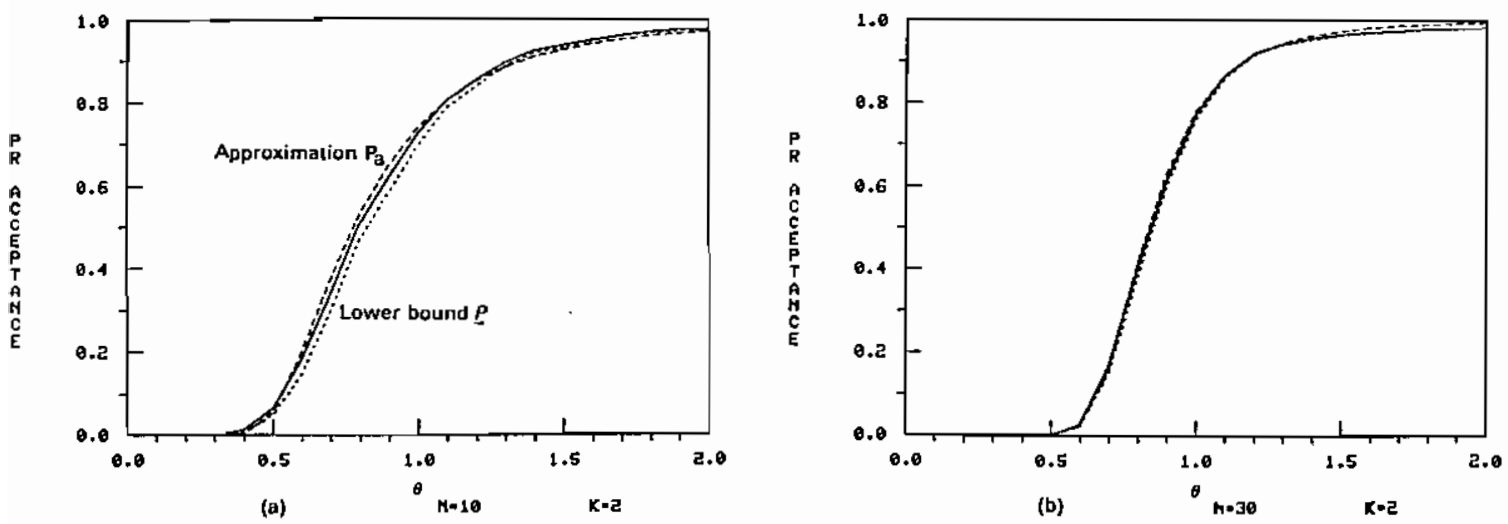

Figure 7. Acceptance probabilities when the number of allowable defectives $k=2$ and $n$ observations are drawn from a Weibull distribution $W(1, \theta)$.
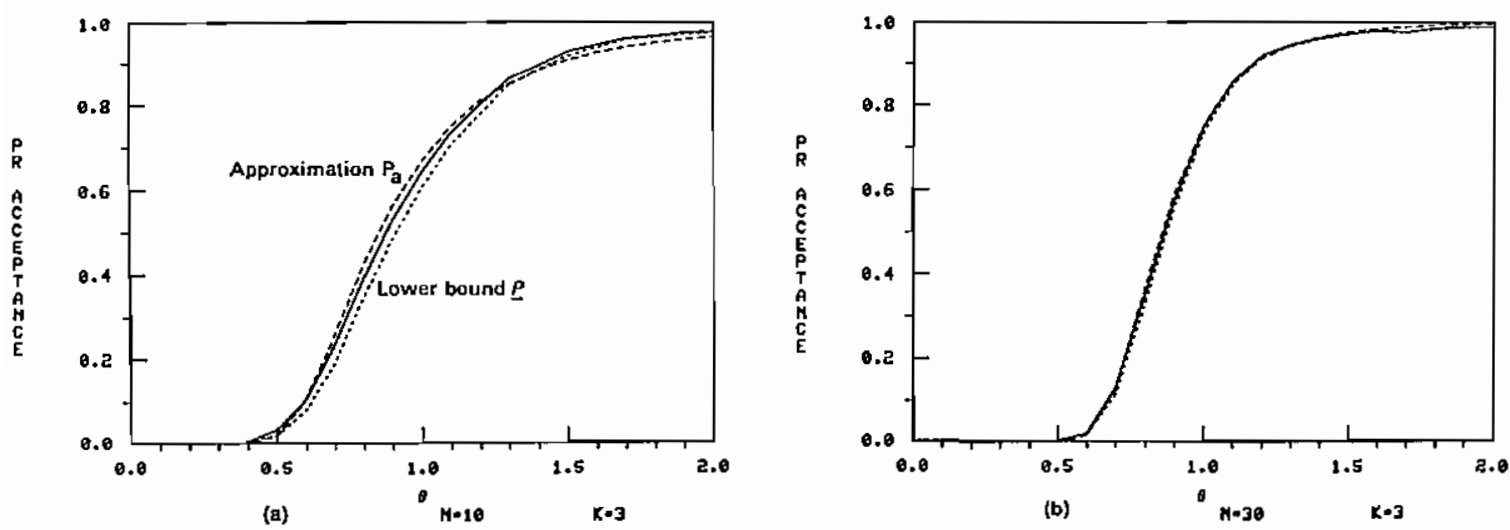

FIGURE 8. Acceptance probabilities when the number of allowable defectives $k=3$ and $n$ observations are drawn Irom a Weibull distribution $W(1, \theta)$.

represented by the dotted line in the same figures. The approximation $P_{a}$ is shown by the heavy dashed line in the figures.

The figures show that $P_{a}$ is not a particularly good approximation to $P_{n}$ when $k=0$, and one would do much better using the lower bound $\underline{P}$. However, $P_{a}$ shows the same characteristie for the Weibull distribution as for the normal distribution; namely, that as $k / n$ increases the accuracy of the approximation increases. For $n=10$ and $k=3, P_{a}$ is superior to $\underline{P}$; for $n=30, \underline{P}$ is indistinguishable from the simulated acceptance probability. 


\subsection{Exponential Distribution}

\subsubsection{Comparison with a UMP Test}

As discussed in section 1 , we may view the problem of finding an optimal sampling procedure as a hypothesis testing problem formulated in (1.4). In general there exists no uniformly most powerful (UMP) test for (1.4). However, it is interesting to note that in the exponential distribution the dual acceptance criterion for $k=0$ corresponds to a test which is UMP for a subset of alternatives specified in (1.4). Specifically, suppose the sample comes from the exponential pdf given in (3.4.1).

The UMP acceptance region for testing

$$
H_{0}: \lambda=\lambda^{*} \text { and } \beta=\beta^{*}
$$

versus

$$
H_{1}: 0<\lambda<\lambda^{*} \text { and } 0<\beta<\beta^{*}
$$

is given by

$$
\left[\bar{X} \geqslant \mu_{0}, X_{(1)} \geqslant \beta^{*}\right]
$$

This testing problem is equivalent to testing

$$
H_{0}: \lambda=\lambda^{*} \text { and } p=p^{*}
$$

versus

$$
H_{1}: 0<\lambda<\lambda^{*} \text { and } p>1-\left(1-p^{*}\right)^{\lambda * / \lambda}
$$

where

$$
p^{*}=1-\exp \left\{-\left(L-\beta^{*}\right) / \lambda^{*}\right\}
$$

or

$$
\beta^{*}=L+\lambda^{*} \log \left(1-p^{*}\right) .
$$

Under $H_{0}, P_{\lambda^{*}, \beta^{*}}\left[X_{(1)} \geqslant \beta^{*}\right]=1$, and $\mu_{0}$ is determined by the equation

$$
P_{\lambda^{*}, \beta^{*}}\left[\bar{X} \geqslant \mu_{0}\right]=1-\alpha,
$$

where $\alpha$ is a predetermined level of significance (Lehmann [15]).

If we set $L=\beta^{*}$ and $k=0$, the test specified by (5.4.1) clearly is the same test specified by (1.3), and the acceptance probability

$$
P_{\mathrm{n}}=P_{\lambda, \beta}\left\{\bar{X} \geqslant \mu_{0}, X_{(1)} \geqslant \beta^{*}\right\}
$$

can be computed either by the approximation shown in section 3.4 or by numerical integration using an exact formula for the distribution of $\bar{X}$ and $N_{L}$ as shown in the next section.

\subsubsection{Exact Distribution of $\bar{X}$ and $N_{L}$}

The joint distribution of $\bar{X}$ and $N_{L}$ can be obtained from the order statistics. 
Let

$$
\begin{aligned}
& Z_{1}=n X_{(1)} \\
& Z_{i}=(n-i+1)\left(X_{(i)}-X_{(i-1)}\right)
\end{aligned}
$$

We have the pdf of $Z_{(1)}$,

$$
g_{1}\left(z_{1}\right)=\lambda^{-1} \exp \left\{-\left(z_{1}-n \beta\right) / \lambda\right\}, z_{1}>\beta
$$

and for $i \geqslant 2, Z_{\mathrm{i}}$ has a pdf

$$
g_{i}\left(z_{i}\right)=\lambda^{-1} \exp \left(-z_{i} / \lambda\right), z_{i} \geqslant 0 .
$$

To compute the acceptance probability $P_{n}$ for an arbitrary k, we make use of the fact that the $Z$ 's are independent r.v.'s, and that

$$
\begin{aligned}
& \sum_{j=1}^{n} X_{\mathrm{j}}=\sum_{j=1}^{n} Z_{j}=\sum_{j=1}^{n} X_{(j)} \text { and proceed as follows: } \\
& P_{n}=P_{\lambda, \beta}\left[\bar{X} \geqslant \mu_{0}, N_{L} \leqslant k\right] \\
& =\int \ddot{A} \int P\left[\sum_{1}^{n} Z_{i} \geqslant n \mu_{0}, \sum_{1}^{k+1} Z_{i} /(n-i+1)>L \mid z_{1}, \cdots, z_{k+1}\right]\left\{\begin{array}{c}
k+1 \\
\pi
\end{array} g_{i}\left(z_{i}\right)\right\} d z_{1} \cdots d z_{k+1} \\
& =\int \ddot{A} \int P\left[\sum_{k+2}^{n} Z_{i} \geqslant n \mu_{0}-\sum_{1}^{k+1} z_{i}\right]\left\{\begin{array}{c}
k+1 \\
1
\end{array} g_{i}\left(z_{i}\right)\right\} d z_{1} \cdots d z_{k+1}
\end{aligned}
$$

where $A=\left\{\left(z_{1}, \cdots, z_{k+1}\right\}: \sum_{1}^{k+1} z_{i} /(n-i+1)>L\right.$ and $n \mu_{0}-\sum_{1}^{k+1} z_{i} \geqslant 0 \mid$.

The expression in (5.4.5) is the exact probability of acceptance, $P_{n}$.

When $k=0$, the computation of $P_{n}$ reduces to

$$
P_{n}=\int_{n L}^{n \mu_{0}} P\left[\sum_{2}^{n} Z_{i} \geqslant n \mu_{0}-z_{1}\right] g_{1}\left(z_{1}\right) d z_{1}+\int_{n \mu_{0}}^{\infty} g_{1}\left(z_{1}\right) d z_{I} .
$$

Note that the sum $Y=\sum_{2}^{n} Z_{i}$ has a gamma density.

$$
f(y)=\frac{(1 / \lambda)^{n-1}}{\Gamma(n-1)} y^{n-2} \exp (-y / \lambda) .
$$

Substituting (5.4.4) and (5.4.7) in (5.4.6) we obtain

$$
P_{n}=\frac{1}{\Gamma(n-1)} \int_{a}^{b} \int_{c}^{\infty} e^{-v} v^{n-2} \exp \left\{-\left(z_{1}-n \beta\right) / \lambda\right\} d v d z_{1}+\exp \left\{-n\left(\mu_{0}-\beta\right) / \lambda\right\}
$$

where

$$
\begin{aligned}
a & =n L \\
b & =n \mu_{0} \\
c & =\left(n \mu_{0}-z_{1}\right) / \lambda
\end{aligned}
$$

The lower bound for $P_{n}$ is

$$
\left.\underline{P}=\int_{2 \pi\left(\mu_{0}-\beta\right) / \lambda}^{\infty} f x\right) d x \int_{2 \pi(L-\beta) / \lambda}^{\infty}
$$


where $f(x)$ is the pdf of the $\chi^{2}(2 n)$ and $g(x)$ is the pdf of the $\chi^{2}(2)$.

\subsubsection{Acceptance probabilities}

If we assume that $X_{1}, \cdots, X_{n}$ are i.i.d. $E(\lambda, 0)$, the acceptance probability for $k=0$

$$
P_{n}=P_{\lambda}\left[\bar{X} \geqslant \mu_{0}, N_{L} \leqslant 0\right]=P\left[\bar{X} \geqslant \mu_{0}, X_{(1)}>L\right]
$$

is computed from (5.4.8) using a numerical integration technique that takes advantage of the fact that the inner integral is an incomplete $\Gamma$-function. Note that $\mu_{0}$ is determined from $\chi^{2}(2)$ according to (5.4.2), and $L$ is determined according to (3.4.5). The acceptance probabihty $P_{n}$ is shown as the solid line in figure 9.
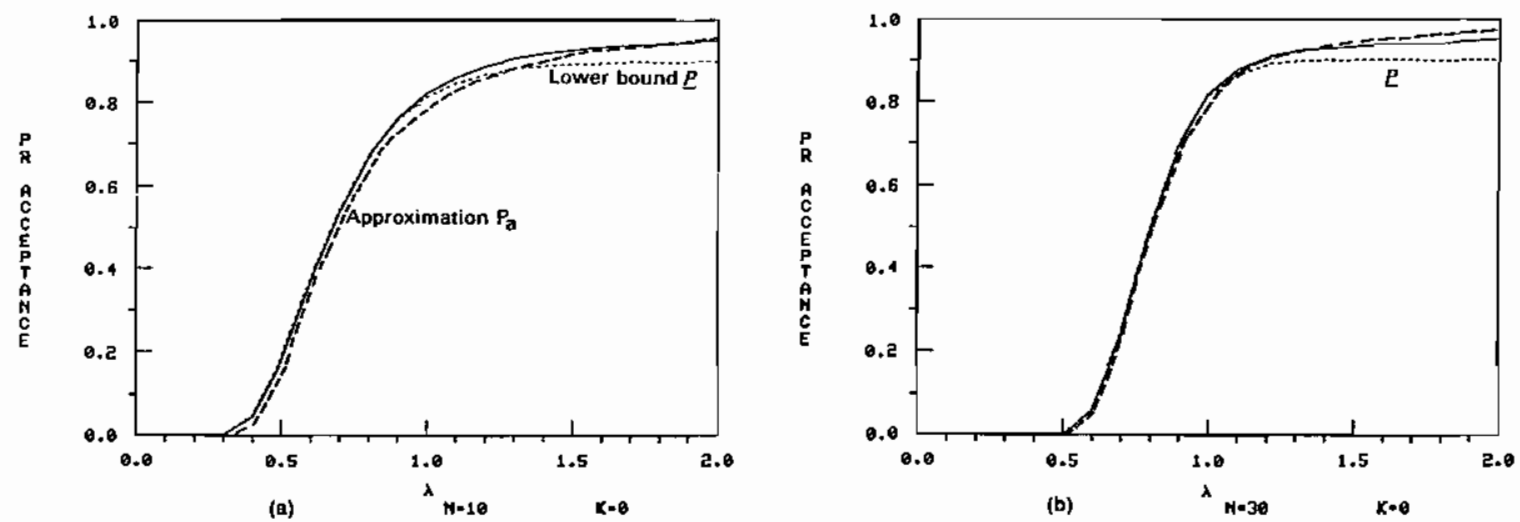

FIGURE 9. Aceeptanee probabilities when the number of allowable defeetives $k=0$ and $n$ observations are drawn from an exponential distributiou $E(\lambda, 0)$.

The acceptance probabilities for $\mathrm{k}=1,2,3$, for $\mu_{0}=0.75$ and $L$ chosen according to (3.4.5) were computed by simulation as were the corresponding values of $\underline{P}$. The approximation $P_{a}$ was computed from (3.4.6). Results are shown in figures 10-12.

The graphs show that $P_{a}$ is a better approximation to $P_{n}$ than the lower bound $\underline{P}$ for small sample size where the superiority of $P_{a}$ over $\underline{P}$ increases as $k$ increases. For large sample size, say $n=30$, the two methods give almost identical approximations to $P_{n}$.

Values of $\mu_{0}$ used in Computation of

Acceptance Probabilities for UMP Tesı for

Exponential Distribution

\begin{tabular}{cccc}
\hline & \multicolumn{3}{c}{ Values of $\mu_{0}$} \\
\hline$n$ & $\alpha=0.10$ & $\alpha=0.05$ & $\alpha=0.01$ \\
5 & 0.48652 & 0.39403 & 0.25582 \\
10 & 0.62213 & 0.64254 & 0.41302 \\
20 & 0.77626 & 0.66273 & 0.55411 \\
30 & 0.77431 & 0.71998 & 0.62475 \\
\hline
\end{tabular}



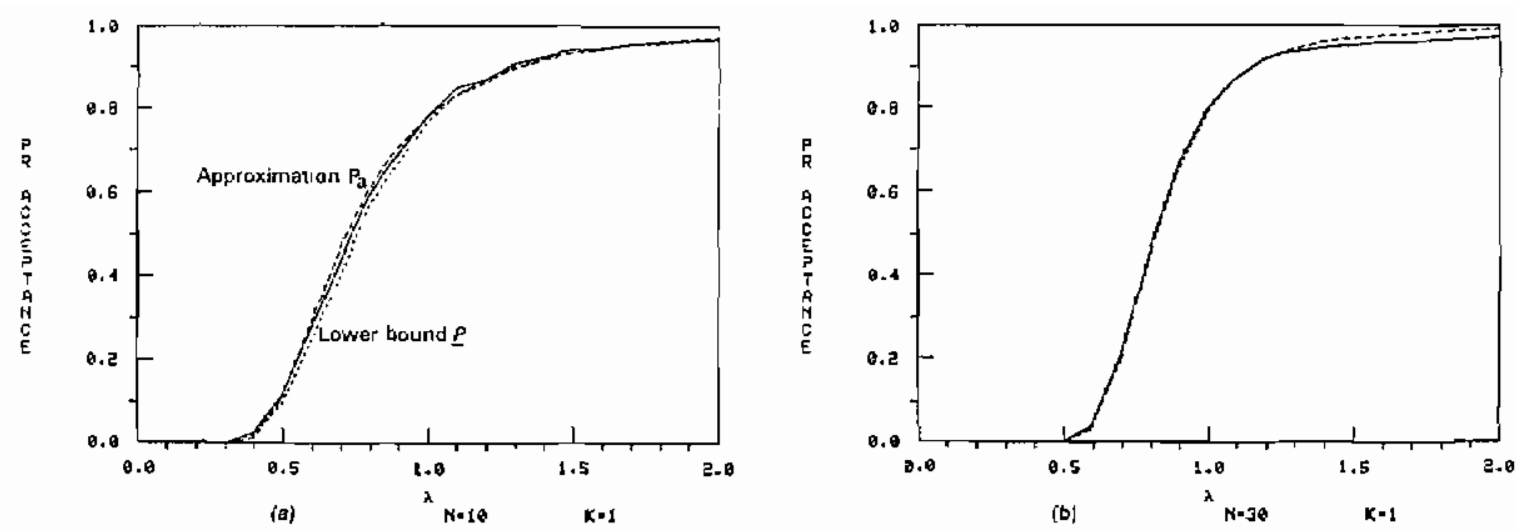

Figure 10. Acceptance probabilities when the number of allowable defectives $k=1$ and $n$ observations are drawn Irom an exponeatial distribution $E(\lambda, 0)$.
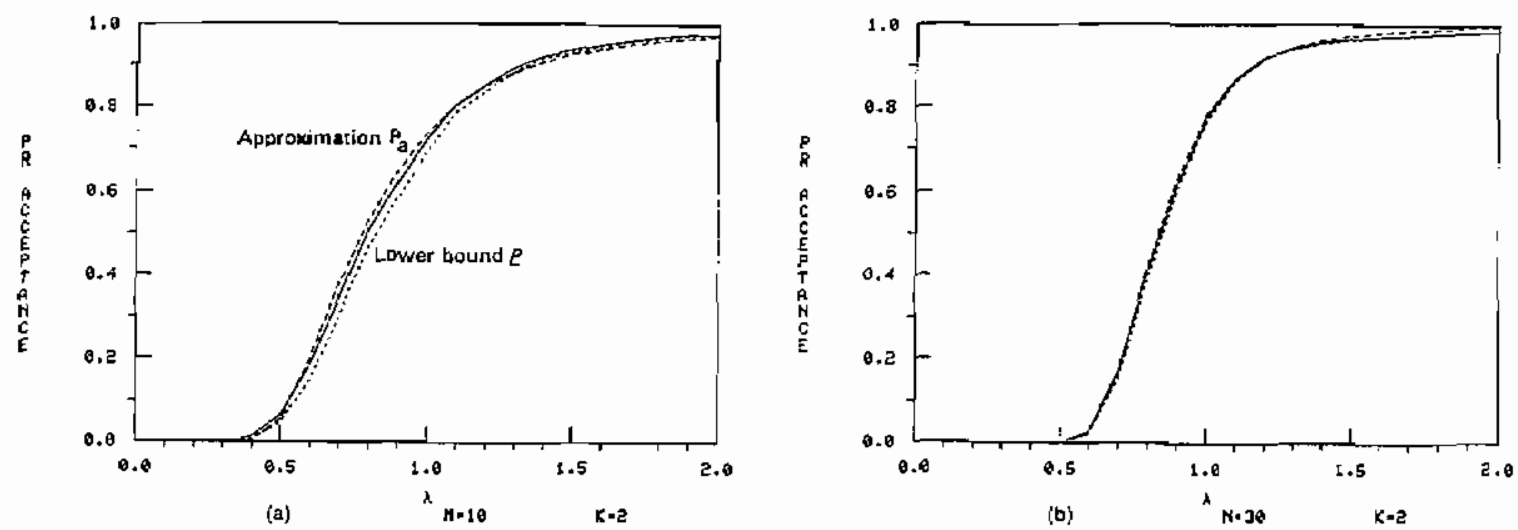

Figune 11. Acceptance probabilities when the number of allowable delectives $k=2$ and $n$ observations are drawn from an exponential distribution $E[\lambda, 0$.
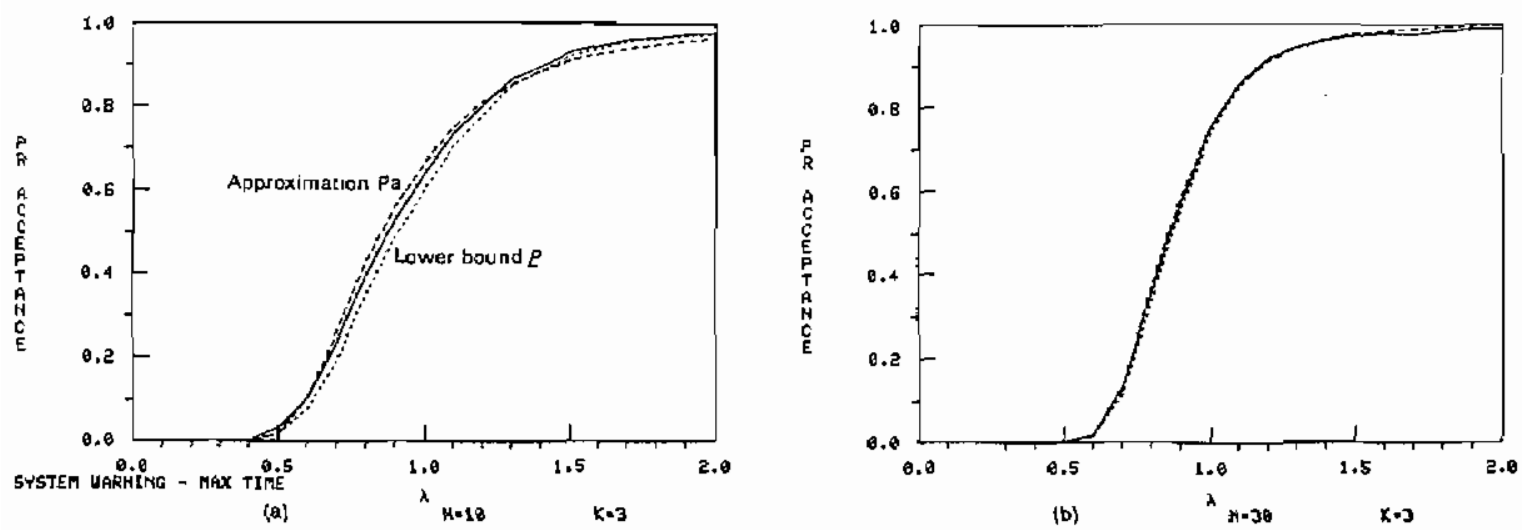

FIGURE 12. Acceptance probabilities when the number of allowable defectives $k=3$ and $n$ observation $\theta$ are drawn from an exponential distribution $E(\lambda, 0)$.

\section{Synopsis}

The problem of computing the acceptance probability $P_{n}$ has been addressed by an approximation $P_{g}$ that relies on the asymptotic joint distribution of the sample mean and number of defectives in the sample. $P_{a}$ has the advantage that it is applicable to any continuous distribution. It is computed using 
a $N(0,1)$ cdf and a bivariate normal cdf which in turn can be reduced to a single variable integration.

The approximation $P_{a}$ compares very favorably with another published approximation for the normal distribution and with a lower bound $P$. Graphs of the acceptance probability as a function of one parameter of the distribution are used to compare the relative accuracies of $P_{a}$ and $\underline{P}$. The graphs show that for the normal distribution $P_{a}$ and $\underline{P}$ have comparable accuracies with $\mathrm{k}=0 . \mathrm{As} k / n$ increases, $P_{a}$ quickly becomes superior to $\underline{P}$, and even for large $\mathrm{n}$ and $k>0 P_{a}$ is superior. In other words, the best results for the normal distribution are obtained with $\underline{P}$ when $\mathrm{k}=0$ and with $P_{a}$ for all other values of $k$.

In the case of Weibull distribution $\underline{P}$ is superior for $k=0$. As $k / n$ increases, $P_{a}$ gains in accuracy, and for large n, $P$ continues to have an edge over $P_{a}$. The difficulty in computing $P$ for the Weibull distribution may make it desirable to use $P_{a}$ for all applications.

In the case of exponential distribution, the exact joint distribution of the sample mean and number of defectives in the sample has been derived for $k=0$. The computation of the acceptance probability $P_{n}$ in this case involves a two-variable integration. Graphs of the acceptance probabilities show that the lower limit $\underline{P}$ gives a consistently good approximation to the acceptance probability. The approximation $\mathbf{P}_{\mathrm{a}}$ and the lower limit $\underline{P}$ have also been computed for the exponential distribution for $1 \leqslant k \leqslant$ 3. The graphs for these tests show that $\underline{P}$ is comparable or superior to $P_{a}$ for large $n(n \geqslant 30)$ with $P_{a}$ being somewhat superior when $n$ is small, say $n \leqslant I 0$.

The numerical integrations for this study were performed using the NBS software package DATAPLOT developed by Dr. J.J. Filliben, and the graphs were prepared using the same package. The authors wish to acknowledge the helpful suggestions for changes in the manuscript made by Dr. P. Smith and Mrs. M. Natrella.

\section{References}

[1] American Society for Testing and Materials. C825-79 Standard Specilications for Preeast Conerete Barrier.

[2] Anderson, T.W. (1958). An Introduction to Multivariate Statistical Analysis. John Wiley \& Sons, Inc: New York.

[3] Box, G.P. and Muller, M.E. (1958). A note on the generation of random normal deviates. JACM. pp. 620-611.

[4] Brickenkamp, C.S., Hasko, S., Natrella, M.G. (1981). Checking the Net Contents of Packaged Goods. NBS Handbook 133. U.S. Government Printing Offiee: Washington. pp. 2-7.

[5] Bronssalian et al. (1975). Considerations in the Use of Sampling Plans for Effecting Complianee with Mandatory Safety Standards. NBSIR 75-697. National Bnreau of Standards, Gaithersburg, MD.

[6] Chnng, K.L. (1960). Markov Chains with Stationary Transition Probabilities. Springer-Verlag: Berlin. p. 84.

[7] Eisenberger, I. (1968). Testing the Mean and Standard Deviation of a Normal Distribution Using Quautiles. Technometrics. 10 (4) pp. 781-91.

[8] Elder, Robert S. and Muse, H. David (1982). An Approximate Method for Evaluating Mixed Sampling Plans. Technometries, 24(3), pp. 207-212.

[9] Environmental Protection Agency (1971). National primary and secondary ambient air quality standards. Federal Register, 36(84). pp. 8186-8201.

[10] Esary, J.D., Proschan, F. and W alkup, D.W. (1967). Association of Random Variables with Applications. Annals of Math Stat, 38. pp. 1466-1474.

[11] Gross, A.J. and Clark, V.A. (1975). Survival Distributions: Reliability Applications in the Biomedical Sciences. John Wiley \& Sons, Inc: New York pp. 14-18.

[12] Harter, H. Leon. (1964). New Tables of the.Incomplete Gamma-Function Ratio and of Percentage Points of the Chisquare and Beta Distributions. US Government Printing Office: Washington.

[13] Johnson, N.L. and Kotz, S. (1970). Continuous Univariate Distributions - 1. Houghton Mifflin Co: Boston. pp. $251-252$.

[14] Lauer, G.N. (1982). Probabilities of Noncompliance for Sampling Plans in NBS Handbook 133. Journal of Quality Tech, 14 (3). pp. 162-165.

[15] Lehmann, E.L. (1959). Testing Statistical Hypotheses, John Wiley \& Sons, Inc.: New York. p. 110.

[16] Pearson, E.S. and Hartley, H.O. (1969). Biometrika Tables for Statisticians, Vol. I (1st edition). University Press: Cambridge. p. 43 .

[17] Perlman, M.D. (1980). Unbiasedness of likelihood ratio tests for equality of several covariance matrices and equality of several multivariate normal populations. Annals of Stat, B(2). pp. 247-263. 
[18] Perng, S.A. (1977). An Asymptotically Efficient Test for the Location Parameter and the Scale Parameter of an Exponenlial Distribution. Comm in Stat. Theory and Methods, A6 (14). pp. 1399-1 407.

[19] Schilling, E.G. and Dodge, H.F. (1969). Procedures and Tables for Evaluating Dependent Mixed Acceptance Sampling Plans. Technometries, 11(2). pp. 341-372.

[20] Tables of the Bivariate Normal Distribution Function and Related Functions. 11959). NBS AMS 50. US Government Printing Office: Washington. pp. vi-vii.

[21] Weed, R.M. (1982). Bounds for Correlated Componnd Probabilities. Journal of Quality Tech. 14(4). pp. $196-200$.

[22] Weed, R.M. (1980). Analysis of Multiple Acceptance Criteria. Trans. Research Record, 745 pp. 20-23.

\section{Appendix A}

The approximation $P_{a}$ given in (3.1.9) involves the computation of $L(a, b, \varrho)$ defined as

$$
L(a, b, \varrho)=\int_{a}^{\infty} \int_{b}^{\infty} g(z, y, \varrho) d y d z .
$$

The computation of $L(a, b, \varrho)$ can be reduced to a single variable integration. When $a$ and $b$ are both positive [18],

$$
L(a, b, \varrho)=\frac{1}{2 \pi} \int_{\operatorname{arc} \cos e}^{\pi} \exp \left\{-\frac{1}{2}\left(a^{2}+b^{2}-2 a b \cos w\right) \operatorname{cosec}^{2} w \mid d w\right.
$$

The following recursion relations hold:

$$
\begin{aligned}
L(-a, b, \varrho) & =-L(a, b,-\varrho)+\frac{1}{2}[1-h(b)] \\
L(a,-b, \varrho) & =-L(a, b,-\varrho)+\frac{1}{2}[1-h(a)] \\
L(-a,-b, \varrho) & =L(a, b, \varrho)+\frac{1}{2}[h(a)+h(b)] \\
\text { where } h(x) & =\int_{-x}^{x} \exp \left(-t^{2} / 2\right) d t .
\end{aligned}
$$

The approximation $P_{a}$ can be computed for all values of $a, b$ and $\varrho$ using the foregoing equations.

$$
\begin{aligned}
& P_{a}=\Phi(-a)-L(a, b, \varrho), a>0, b>0 \\
& P_{a}=\Phi(-a)-\Phi(-b)+L(-a, b,-\varrho), a<0, b>0 \\
& P_{a}=L(a,-b,-\varrho), a>0, b<0 \\
& P_{a}=\Phi(b)-L(-a,-b, \varrho), a<0, b<0
\end{aligned}
$$

where $\Phi(x)=\int_{-\infty}^{x} \exp \left(-t^{2} / 2\right) d t$.

\section{Appendix B}

Asymptotic independence of the sample mean and the $(n-k)^{\text {th }}$ extremc statistic.

Let $X_{1}, \cdots, X_{n}$ be i.i.d with a p.d.f. $f(x)$. Denote the c.d.f. of the $X$ 's by $F(x)$. Assume that $X$ 's have a finite mean $\mu$ and finite variance $\sigma^{2}$. Let $X_{(1)}<\cdots<X_{(n)}$ be the order statistics.

The conditional density of $X_{(1)}, \cdots, X_{(n)}$ given that $X_{(n-k)}=x_{(n-k)}$ is given by 


$$
L_{x_{(n-k)}}=\frac{\begin{array}{c}
n-k-1 \\
(n-k-1) ! \Pi f\left(x_{(i)}\right)
\end{array}}{\left\{F\left(x_{(n-k)}\right)\right\}^{n-k-1}} \cdot \frac{k ! \prod_{n-k+1}^{n} f\left(x_{(i)}\right)}{\left\{1-F\left(x_{(n-k)}\right)\right\}}
$$

Clearly, given that $X_{(n-k)}=x_{(n-k)}$, the joint conditional density may be regarded as the joint density of two dependent samples $\left\{Y_{1}, \cdots, Y_{n-k-1}\right\}$ and $\left\{W_{1}, \cdots, W_{k}\right\}$, where the $Y$-sample has a p.d.f.

$$
\begin{aligned}
& h(x)=\frac{f(x)}{F\left(x_{(n-k)}\right)} \text {, if } x<x_{(n-k)} \\
& =0 \quad \text {, if } x>x_{(n-k)}
\end{aligned}
$$

and the $W$-sample has a p.d.f.

$$
\begin{aligned}
g(x) & =\frac{f(x)}{1-F\left(x_{(n-k)}\right)}, & \text { if } x>x_{(n-k)} \\
& =0 & , \text { if } x<x_{(n-k)}
\end{aligned}
$$

THEOREM. For every fixed $k, \sqrt{n}(\overline{\mathrm{X}}-\mu)$ is asymptotically independent of $\mathrm{X}_{(\mathrm{n}-\mathrm{k})}$ as $\mathrm{n} \rightarrow \infty$.

Proof: Rewrite $\bar{X}$ in terms of the $Y$ 's and the $W$ 's. We obtain

$$
\frac{\sqrt{n}(\bar{X}-\mu)}{\sigma}=\sqrt{n-k-1} \frac{(\bar{Y}-\mu)}{\sigma} \frac{\sqrt{n-k-1}}{\sqrt{n}}+\frac{(\bar{W}-\mu) k}{\sigma \sqrt{n}}+\frac{X_{(n-k)}-\mu}{\sigma \sqrt{n}}
$$

From (2) we have

$$
E Y_{i}-\mu=\frac{\int_{0}^{x_{(n-k)}} x d F(x)}{\int_{x_{(n-k)}}^{\infty} d F(x)}-\int_{x_{(n-k)}}^{\infty} x d F(x)
$$

Making use of (4) and (5), and letting $A$ be the value of $E Y_{i}$ with $X_{(n-k)}$ replaced by $X_{(n-k)}$, we get

$$
\begin{gathered}
\frac{\sqrt{n}(\bar{X}-\mu)}{\sigma}=\sqrt{n-k-1} \frac{\left(\bar{Y}-E Y_{i}\right)}{\sigma} \frac{\sqrt{n-k-1}}{\sqrt{n}} \\
+\frac{(n-k-1)(\mathrm{A}-\mu)}{\sqrt{n} \sigma}+\frac{(\bar{W}-\mu)}{\sigma} \frac{k}{\sqrt{n}}+\frac{\mathrm{X}_{(n-k)-\mu}}{\sigma \sqrt{n}}
\end{gathered}
$$

Since $k$ is fixed, clearly $(\bar{W}-\mu) k / \sigma \sqrt{n \rightarrow 0}$ in probability as $n \rightarrow \infty$. To prove the theorem we need the following two lemmas in which we show that the second and the fourth terms tend to zero in probability. Then the theorem follows from the fact that the first term converges in distribution to $N(0,1)$ which is the "unconditional" limiting distribution of $\sqrt{n}(\bar{X}-\mu)$. 
LEMMA 1. As $\mathrm{n} \rightarrow \infty$,

$$
\frac{\mathrm{X}_{(\mathbf{n}-\mathbf{k})}}{\sqrt{\mathrm{n}}} \rightarrow 0 \text { in } \mathrm{P}
$$

Proof: For every $\varepsilon>0$ and for a fixed $k$, it follows from the Chebyehev inequality that

$$
P\left\{\frac{\left|X_{(n-k)}\right|}{\sqrt{n}}>\varepsilon\right\} \leqslant \frac{E\left(X_{(n-k)}\right)^{2}}{n \varepsilon^{2}} \leqslant \frac{1}{n \varepsilon} \quad E\left(\max _{1 \leqslant j \leqslant n} X_{j}^{2)}\right.
$$

Let $Y_{j}=X_{j}^{2}$ and $H(y)=P\left[Y_{j} \geqslant y\right]$.

Following a proof in Chung (1960),

$$
P\left[\max _{1 \leqslant j \leqslant n} Y_{j} \geqslant y\right]=1-[H(y)]^{n} \geqslant n[1-H(y)]
$$

and

$$
\underset{1 \leqslant j \leqslant n}{\frac{1}{n} E\left(\max _{j} X_{j}{ }^{2} \mid=\frac{i}{n}\right.} \int_{0}^{\infty}\left\{1-[H(y)]^{n}\right\} d y \geqslant \int_{0}^{\infty}[1-H(y)] d y<\infty
$$

On the other hand,

$$
\left.\frac{1}{n} E \underset{1 \leqslant j \leqslant n}{E(\max } X_{j}^{2}\right]=\int_{0}^{\infty} \int_{H(y)}^{1} u^{n-1} d u d y
$$

Sinee the expectation is finite, we can take the limit as $n \rightarrow \infty$ under the integral sign. As a result

$$
\lim _{n \rightarrow \infty} \frac{1}{n} E\left[\max _{1 \leqslant j \leqslant n} X_{j}^{2}\right]=\int_{0}^{\infty} \int_{H(y)}^{1} \lim _{n \rightarrow \infty} u^{n-1} d u d y=0
$$

LEMMA 2. For a fixed $k, 0 \leqslant k \leqslant n-1$,

$$
\sqrt{n-\mathrm{k}-\mathrm{l}} \int_{\mathrm{X}_{(\mathrm{n}-\mathrm{k})}}^{\infty} \mathrm{xdF}(\mathrm{x}) \rightarrow 0 \text { in } \mathrm{P} \text { as } \mathrm{n} \rightarrow \infty
$$

Proof: Since

$$
\begin{gathered}
\sqrt{n-k-1} \quad \int_{X_{(n-k)}}^{\infty} x d F(x)=\sqrt{n-k-1} X_{(n-k)}\left[1-F\left(X_{(n-k)}\right]\right. \\
+\sqrt{n-k-1} \quad \int_{X_{(n-k)}}^{\infty}[1-F(x)] d x
\end{gathered}
$$

we will show that each term on the right side of (8) converges in probability to zero. 
Set

$$
z=1-\frac{x}{n-k-1}
$$

Then

$$
P\left\{(n-k-1)\left[1-F\left(X_{(n-k)}\right)\right]>x\right\}=\sum_{i=n-k}^{n}\left(\begin{array}{c}
n \\
i
\end{array}\right) z^{i}(1-z)^{n-i} \rightarrow e^{-x} \sum_{i=0}^{k} \frac{x^{i}}{i !}
$$

We see that $X_{(n-k)} / \sqrt{n-k-1} \rightarrow 0$ in $P$ as shown in Lemma 1 and $(n-k-1)\left[1-F\left(X_{(n-k)}\right)\right]$ converges in distribution as shown in (10). Thus, the first term on the right side of (8) tends to zero in $\underline{P}$.

Finally, to show that the last term in (8) tends to zero in $P$, write this term as

$$
\sqrt{n-k-1} \int_{X_{(n-k)}}^{\infty}[1-F(X)] d x=\sqrt{n-k-1)\left(1-F\left(X_{(n-k)}\right)\right.} \quad\left\{\begin{array}{l}
\infty \\
\int_{X_{(n-k)}}([1-F(x)] d x) / \sqrt{1-F\left(X_{(n-k)}\right)}
\end{array}\right\}
$$

Clearly, the part in braekets tends to zero in $P$ ean be seen by the application of the L'Hospitals's rule to it. 